Publ. RINIS, Kyoto Univ.

13 (1977), (587-7'?')

\title{
On Some Intuitionistic Modal Logics
}

Dedicated to Professor Shigeru Furuya on his 60th Birthday

By

Hiroakira ONO*

\section{Introduction}

Some modal logics hased on logics weaker than the classical logic have been studied by Fitch [4], Prior [18], Bull [1], [2], [3], Prawitz [17] etc. In this paper, we treat modal logics based on the intuitionistic propositional logic, which we call intuitionistic modal logics (abbreviated as IML's). Our main concern is to compare properties of several IML's of S4- or S5-type by using some model theoretical methods. The study of modal logics based on weak logics seems to reveal to us various properties of classical modal logics, especially of S5, which will be indistinguishable by dealing them only on the classical logic.

We will introduce some IML's in the Hilbert-style formalization in $\S 2$. Then we will define IML's in the form of sequent calculi, all of which are given by restricting or modifying the sequent calculi S4 and S5 of Ohnishi-Matsumoto [15]. We will show the proper inclusion relationship between these IML's by using a kind of algebraic models. In $\S \S 3$ and 5, we will introduce two kinds of models for IML's. One of them is a natural extension of Kripke models for the intuitionistic logic and the other is for modal logics (see [11], [12]). Then we will prove the completeness theorem with respect to these models. In $\S 4$, the finite model property for some IML's will be shown.

We would like to thank $M$. Sato for his valuable suggestions.

\section{§ 2. Intuitionistic Modal Logics}

We will introduce some intuitionistic modal logics. We take $\wedge, \vee$,

Communicated by S. Takasu, November 11, 1976.

* College of Integrated Arts and Science`, Hiroshima University, Hiroshima 730, Japan. 
$\supset$, $\neg$ and $\square$ (necessity operator) as primitive. We don't use $\diamond$ (possibility operator), only for brevity's sake. Note that, unlike classical modal logics, $\diamond A$ can not be considered as the abbreviation of $\neg \square \neg A$. But by a technical reason some IML's containing $\diamond$ are treated later.

Let $\mathrm{H}$ be the intuitionistic propositional logic formulated in the Hilbert-style. The rules of inference of $\mathrm{H}$ are modus ponens and the rule of substitution. The IML $\mathrm{L}_{0}$ is obtained from $\mathrm{H}$ by adding the following three axioms,

(1) $\square p \supset p$,

(2) $\square p \supset \square \square p$,

(3) $\square(p \supset q) \supset(\square p \supset \square q)$,

and the rule of necessitation, i.e, from $A$ infer $\square A$. Clearly, $\mathrm{L}_{0}$ with the law of excluded middle $(p \vee \neg p)$ becomes S4. Next, we consider the following axioms;

$$
\begin{aligned}
& A_{1}: \neg \square p \supset \square \neg \square p, \\
& A_{2}:(\square p \supset \square q) \supset \square(\square p \supset \square q), \\
& A_{3}: \square(\square p \vee q) \supset(\square p \vee \square q), \\
& A_{4}: \square p \vee \square \neg \square p .
\end{aligned}
$$

The logic $\mathrm{L}_{0}$ with the axiom $A_{i}$ is denoted by $\mathrm{L}_{i}$ for $i=1,2,3,4$. The logic $\mathrm{L}_{3}$ with $A_{1}$ (or $A_{2}$ ) is denoted by $\mathrm{L}_{31}$ (or $\mathrm{L}_{32}$, respectively). It is easy to see that $\mathrm{S} 4$ with any one of $A_{i}$ is equal to S5. So, we can say that $\mathrm{L}_{0}$ is of S4-type and others are of S5-type. We remark that the logic $\mathrm{L}_{0}$ with either $\neg p \supset \square \neg \square p$ or $p \supset \square \neg \square \neg p$ is equal to $\mathrm{L}_{1}$, and $\mathrm{L}_{0}$ with $\neg \square \neg \square p \supset \square p$ is equal to $\mathrm{L}_{4}$. We can show that $\mathrm{I}_{S 4}$ (or $\mathrm{I}_{S 5}$ ) in [17] is equivalent to $L_{0}$ (or $L_{1}$, respectively) and that MIPC in [18], which contains $\diamond$ as primitive, is a conservative extension of $L_{2}$ as proved in $\S 4$.

We sometimes identify a logic $\mathrm{L}$ with the set of formulas provable in L. If $\mathrm{L}$ and $\mathrm{L}^{\prime}$ are IML's then the set $\mathrm{L} \cap \mathrm{L}^{\prime}$ is closed under modus ponens, the rule of substitution and the rule of necessitation. So $L \cap L^{\prime}$ is also considered as an IML. But the union of $\mathrm{L}$ and $\mathrm{L}^{\prime}$ is not necessarily closed under these rules. Hence, we write $L \cup L^{\prime}$ for the minimum 
set of formulas which contains the union of $\mathrm{L}$ and $\mathrm{L}^{\prime}$ and is closed under these three rules. Then, $L \cup L^{\prime}$ is an IML. In Figure 2.1, we show the lattice made up of our IML's. The proper inclusion relationship between two logics is represented by the line connecting them.

The inclusion relationship shown in Figure 2.1 are almost trivial. It is proved in the last part of this $\S$ that these inclusions are proper.

As another way of introducing IML's, we take the formulation by sequent calculi. We modify the sequent calculi of modal logics S4 and S5 in [15]. Consider the sequent calculi

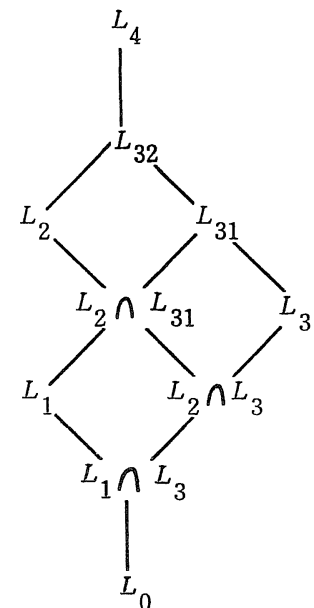

Figure 2.1. obtained from the propositional part of LJ of Gentzen [6] by adding the rules concerning $\square$ of the following form;

$$
(\square \rightarrow) \frac{A, \Gamma \rightarrow \Delta}{\square A, \Gamma \rightarrow \Delta} \quad(\rightarrow \square) \frac{\Gamma \rightarrow A}{\Gamma \rightarrow \square A},
$$

where $\Delta$ consists of at most one formula. In the application of $(\rightarrow \square)$, we impose one of the following conditions on $\Gamma$.

(0) $\Gamma$ is a sequence of formulas of the form $\square B$.

(1) $\Gamma$ is a sequence of formulas of the form $\square B$ or $\neg \square B$.

(2) $\Gamma$ is a sequence of completely modalized formulas, where a formula is said to be completely modalized (abbreviated as c.m.) if any occurrence of a propositional variable in it is within the scope of a necessity operator.

The rule $(\rightarrow \square)$ under the condition (i) is denoted by ( $\rightarrow \square i$ ) for $i=0,1,2$. The sequent calculus $\mathrm{K}_{i}$ is the propositional part of $\mathrm{LJ}$ with $(\square \rightarrow)$ and $(\rightarrow \square i)$.

$\mathrm{LJ}^{\prime}$ is the sequent calculus obtained from LK by restricting applications of rules $(\rightarrow \neg),(\rightarrow \supset)$ and $(\rightarrow \forall)$ to the case where in the lower sequent of the application, only one formula occurs in the succedent. It is known that $\mathrm{LJ}^{\prime}$ is equivalent to $\mathrm{LJ}$ and is cut-free (i.e, the cutelimination theorem for $\mathrm{LJ}^{\prime}$ holds). See [22]. For $i=0,1,2$, let $\mathrm{G}_{i}$ be the sequent calculus obtained from the propositional part of $\mathrm{LJ}^{\prime}$ by 
adding $(\square \rightarrow)$ and $(\rightarrow \square i)$. Then it is easy to see that for each $i, G_{i}$ is equivalent to $\mathrm{K}_{i}$.

Now consider the sequent calculi obtained from the propositional part of $\mathrm{LJ}^{\prime}$ by adding the following rules;

$$
(\square \rightarrow+) \frac{A, \Gamma \rightarrow \Delta}{\square A, \Gamma \rightarrow \Delta} \quad(\rightarrow \square+) \frac{\Gamma \rightarrow \Delta, A}{\Gamma \rightarrow \Delta, \square A} .
$$

In the application of $(\rightarrow \square+)$, we also impose one of the following conditions.

(0) Both $\Gamma$ and $\Delta$ are sequences of formulas of the form $\square B$.

(1) Both $\Gamma$ and $\triangle$ are sequences of formulas of the form $\square B$ or $\neg \square \mathrm{B}$.

(2) Both $\Gamma$ and $\Delta$ are sequences of c.m. formulas.

The rule $(\rightarrow \square+)$ under the condition (i) is denoted by $(\rightarrow \square+i)$ for $i=0,1,2$. The sequent calculus $\mathrm{G}_{3}\left(\mathrm{G}_{31}, \mathrm{G}_{32}\right)$ is the propositional part of $\mathrm{LJ}^{\prime}$ with $(\square \rightarrow+)$ and $(\rightarrow \square+0)$ (or $(\rightarrow \square+1)$ or $(\rightarrow \square+2)$, respectively).

The sequent calculus $G_{4}$ is obtained from $G_{3}$ by replacing the rules $(\rightarrow \neg)$ and $(\rightarrow \supset)$ by the following rules $\left(\rightarrow \neg^{*}\right)$ and $\left(\rightarrow \supset^{*}\right)$.

$$
(\rightarrow \neg *) \frac{A, \Gamma \rightarrow \Delta}{\Gamma \rightarrow \Delta, \neg A}\left(\rightarrow \supset^{*}\right) \frac{A, \Gamma \rightarrow \Delta, B}{\Gamma \rightarrow \Delta, A \supset B},
$$

where $\Delta$ is a sequence of formulas of the form $\square C$. (The definition of $\mathrm{G}_{4}$ mentioned above is suggested by Sato.)

Theorem 2. 1. For $J=0,1,2,3,31,32,4, G_{J}$ is equivalent to $L_{J}$, i.e. for any formula $A, A$ is provable in $G_{J}$ if and only if $A$ is provable in $L_{J}$.

We will prove in $\S 3$ that $\mathrm{K}_{0}$ and $\mathrm{G}_{0}$ are cut-free. On the other hand, we can show that others are not cut-free. For, the formula $P \supset$ $\square \neg \square \neg p$ is provable in $\mathrm{G}_{1}$ (and $\mathrm{K}_{1}$ ) and hence in $\mathrm{G}_{2}$ (and $\mathrm{K}_{2}$ ), but is not provable without cut in any of them. Also, $\square(\square p \vee q) \supset \square p \vee \neg \neg \square q$ is provable in $\mathrm{G}_{3}, \mathrm{G}_{31}$ and $\mathrm{G}_{32}$, but is not provable without cut in any of them. The sequent $\rightarrow p, \square \neg \square p \quad$ is provable in $\mathrm{G}_{4}$ but is not provable without cut in it. Henceforth, we sometimes write $G \vdash \Gamma \rightarrow \Delta$ if $\Gamma \rightarrow \Delta$ 
is provable in the sequent calculus $\mathrm{G}$.

We introduce algebraic models for IML's, which correspond to topological Boolean algebras in classical modal logics. A topological pseudoBoolean algebra (abbreviated as a $\iota p b a$ ) is a pair $(P, I)$ of a nondegenerate pseudo-Boolean algebra $P$ and a unary operation $I$ on $P$ such that for each $a, b \in P$,

i) $\quad I(a \cap b)=I a \cap I b$,

ii) $\quad$ I $a \leq a$,

iii) $\quad I I a=I a$,

iv) $\quad I 1=1$,

where 1 is the greatest element of $P$. An element $a$ in $P$ is said to be open in a tpba $(P, I)$, if $I a=a$ holds. We can see that the set of all open elements in $(P, I)$ constitutes a sublattice of $P$. An assignment of a tpba $(P, I)$ is defined in the usual way. In particular, for any assignment $f, f(\square A)=I f(A)$. A formula $A$ is valid in a tpba $(P, I)$ if $f(A)=1$ for any assignment $f$ of $(P, I)$.

We define tpba's of type $J$ for $J=0,1,2,3,31,32,4$ as follows.

0) Any tpba is of type 0 .

1) A tpba is of type 1 if the complement of any open element in it is also open.

2) A tpba $(P, I)$ is of type 2 if the set of all open elements in it constitutes a sub-(pseudo-Boolean) algebra of $P$.

3) A tpba is of type 3 if it satisfies the condition

v) $\quad I(I a \cup b) \leq I a \cup I b$.

3i) For $i=1,2$, a tpba is of type $3 i$ if it is of type 3 and also of ty'pe $i$.

4) A tpba is of type 4 if it is of type 2 and the subalgebra constituted by the set of all open elements is a Boolean algebra.

We remark that in any tpba, $I a \cup I b \leq I(I a \cup b)$ holds. By using the Lindenbaum algebra, we get the following theorem.

Theorem 2. 2. For $J=0,1,2,3,31,32,4$, a formula is provable in $L_{J}$ if and only if it is valid in any tpba of type $J$. 
Next we show that there exist uncountably many IML's of S5-type, by using the McKinsey-Tarski translation [14]. Let $T$ be a translation from the set of formulas of propositional logics to the set of formulas of model logics such that

1) $T(p)=\square p$ if $p$ is a propositional variable,

2) $\quad T(A \wedge B)=T(A) \wedge T(B)$,

3) $\quad T(A \vee B)=T(A) \vee T(b)$,

4) $T(A \supset B)=\square(T(A) \supset T(B))$,

5) $\quad T(\neg A)=\square \neg T(A)$.

Let $\mathrm{C}$ be the classical propositional logic. An intermediate propositional logic is a set of formulas, which contains $\mathrm{H}$ and is contained by $\mathrm{C}$ and which is closed under modus ponens and the rule of substitution. Let $J$ be any one of $0,1,2,3,31,32,4$. For any intermediate propositional logic $\mathrm{L}, T_{J}(\mathrm{~L})$ denotes the IML obtained from $\mathrm{L}_{J}$ by adding every formula in $\{T(A) ; A$ is in $\mathrm{L}\}$ as axioms.

Lemma 2. 3. For every intermediate propositional logics $L, L^{\prime}$,

1) $L \subseteq L^{\prime}$ implies $T_{J}(L) \subseteq T_{J}\left(L^{\prime}\right)$,

2) i. $T_{J}\left(L \cap L^{\prime}\right)=T_{J}(L) \cap T_{J}\left(L^{\prime}\right)$, ii. $\quad T_{J}\left(L \cup L^{\prime}\right)=T_{J}(L) \cup T_{J}\left(L^{\prime}\right)$,

3) $L \neq L^{\prime}$ implies $T_{J}(L) \neq T_{J}\left(L^{\prime}\right)$ if $J \neq 4$.

Proof. 1) and 2) are obvious. We prove 3). Suppose that $\mathrm{L} \neq \mathrm{L}^{\prime}$ and $A \in \mathrm{L}-\mathrm{L}^{\prime}$. Then there exists a pseudo-Boolean algebra $P$, in which 1) every formula provable in $\mathrm{L}^{\prime}$ is valid and 2) $A$ is not valid. Define a unary operation $I$ on $P$ by $I a=a$ for any $a \in P$. Then $(P, I)$ is a tpba, in which 1) every formula provable in $T_{J}\left(\mathrm{~L}^{\prime}\right)$ is valid and 2) $T(A)$ is not valid. So, $T(A) \notin T_{J}\left(\mathrm{~L}^{\prime}\right)$. On the other hand, $T(A) \in T_{J}(\mathrm{~L})$. Thus, $T_{J}(\mathrm{~L}) \neq T_{J}\left(\mathrm{~L}^{\prime}\right)$.

We remark that 1) for $J=2,32,4, T_{J}(\mathrm{H})=\mathrm{L}_{J}$ and 2) $T_{0}(\mathrm{C})$ $=T_{4}(\mathrm{C})=\mathrm{L}_{4}$. Jankov proved in [9] that there exist uncountably many 
intermediate propositional logics. So by Lemma 2.3, we have the following.

Corollary 2. 4. There are uncountably many IML's between $L_{2}$ (or $L_{32}$ ) and $L_{4}$.

Theorem 2.5. 1) Following three conditions are equivalent.

i. A is provable in $H$.

ii. $T(A)$ is provable in $T_{0}(H)$.

iii. $T(A)$ is provable in $S 4$.

2) Following three conditions are equivalent.

i. A is provable in $C$.

ii. $T(A)$ is provable in $L_{4}$.

iii. $T(A)$ is provable in $S 5$.

Proof. In each case, it is well-known that i. is equivalent to iii. Clearly, i. implies ii. and ii. implies iii.

Finally, we show that each inclusion relationship shown in Figure 2.1 is proper。

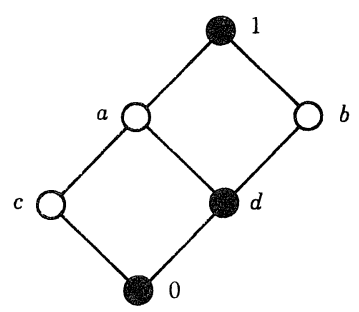

Figure 2.?.

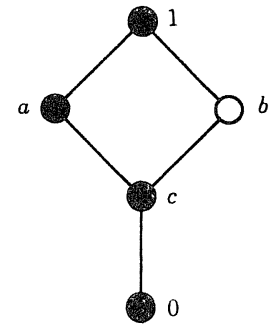

ligure 2.3.

Lemma 2. 6. It holds that 1) $\left.L_{3} \subsetneq L_{31}, 2\right) \quad L_{1} \cap L_{3} \subsetneq L_{1}$ and 3) $L_{2} \cap L_{3} \subsetneq L_{2} \cap L_{31}$.

Proof. Consider the tpba $\left(P_{1}, I_{1}\right)$ shown in Figure 2.2, in which it holds $I_{1} a=I_{1} b=I_{1} d=d$ and $I_{1} c=0$. In the figure, we indicate an open 
element by a black dot. It is easy to see that $\left(P_{1}, I_{1}\right)$ is a tpba of type 3 . We show that $A_{1}$ is not valid. Let $f(p)=d$. Then,

$$
f\left(A_{1}\right)=f(\neg \square p \supset \square \neg \square p)=c \supset 0=b \neq 1 \text {. }
$$

Thus, $\mathrm{L}_{1} \nsubseteq \mathrm{L}_{3}$. Hence $\mathrm{L}_{3} \subsetneq \mathrm{L}_{31}=\mathrm{L}_{1} \cup \mathrm{L}_{3}$ and $\mathrm{L}_{1} \cap \mathrm{L}_{3} \subsetneq \mathrm{L}_{1}$. Assume that $\mathrm{L}_{2}$ $\cap \mathrm{L}_{3}=\mathrm{L}_{2} \cap \mathrm{L}_{31}$. Since $\mathrm{L}_{2} \cap \mathrm{L}_{31}=\mathrm{L}_{2} \cap\left(\mathrm{L}_{1} \cup \mathrm{L}_{3}\right)=\mathrm{L}_{1} \cup\left(\mathrm{L}_{2} \cap \mathrm{L}_{3}\right), \mathrm{L}_{1} \subseteq \mathrm{L}_{2} \cap \mathrm{L}_{3}$ holds. But this implies $\mathrm{L}_{1} \subseteq \mathrm{L}_{3}$. This is a contradiction. Hence $\mathrm{L}_{2} \cap \mathrm{L}_{3}$ $\subsetneq \mathrm{L}_{2} \cap \mathrm{L}_{31}$.

Lemma 2.7. It holds that 1) $L_{1} \subsetneq L_{2} \cap L_{31}$ and 2) $L_{1} \cap L_{3} \subsetneq L_{2}$ $\cap L_{3}$.

Proof. Consider the tpba $\left(P_{2}, I_{2}\right)$ in Figure 2. 3, in which $I_{2} b=c$ holds. It can be verified that $\left(P_{2}, I_{2}\right)$ is of type 1 . We show that $A_{2} \vee A_{3}^{\prime}$ is not valid in it, where $A_{3}^{\prime}$ is $\square(\square r \vee s) \supset(\square r \vee \square s)$. Define an assignment $f$ by $f(p)=f(r)=a$ and $f(q)=f(s)=b$. Then,

$$
\begin{aligned}
f\left(A_{2} \bigvee A_{3}^{\prime}\right) & =\left((a \supset c) \supset I_{2}(a \supset c)\right) \cup\left(I_{2}(a \cup b) \supset(a \cup c)\right) \\
& =(b \supset c) \cup(1 \supset a) \\
& =a \neq 1 .
\end{aligned}
$$

Since $A_{2} \bigvee A_{3}^{\prime}$ is a theorem of $\mathrm{L}_{2} \cap \mathrm{L}_{3}, \mathrm{~L}_{2} \cap \mathrm{L}_{3} \not \mathrm{L}_{1}$. Now suppose that $\mathrm{L}_{1}=\mathrm{L}_{2} \cap \mathrm{L}_{31}$. Since $\mathrm{L}_{2} \cap \mathrm{L}_{31}=\mathrm{L}_{2} \cap\left(\mathrm{L}_{1} \cup \mathrm{L}_{3}\right)=\mathrm{L}_{1} \cup\left(\mathrm{L}_{2} \cap \mathrm{L}_{3}\right), \mathrm{L}_{2} \cap \mathrm{L}_{3} \subseteq \mathrm{L}_{1}$ by the assumption. But this is a contradiction. Hence 1) holds. 2) is easily derived from 1 ).

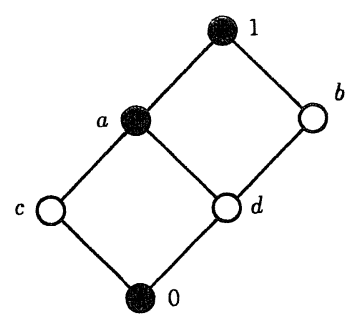

Figure 2.4.

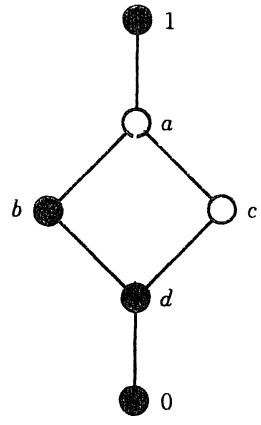

Figure 2.5.

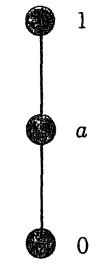

Figure 2.6. 
Lemma 2. 8. It holds that 1) $L_{2} \subsetneq L_{32}$, 2) $L_{2} \cap L_{31} \subsetneq L_{31}$ and 3) $L_{2} \cap L_{3} \subsetneq L_{3}$.

Proof. Consider the tpba $\left(P_{3}, I_{3}\right)$ in Figure 2.4. Notice that $I_{3} b$ $=I_{3} c=I_{3} d=0$. It is easily seen that $\left(P_{3}, I_{3}\right)$ is of type 2. Let $f(p)=a$ and $f(q)=b$. Then,

$$
f\left(A_{3}\right)=I_{3}\left(I_{3} a \cup b\right) \supset\left(I_{3} a \cup I_{3} b\right)=1 \supset a=a \neq 1 .
$$

Thus, $A_{3}$ is not valid. Hence, $\mathrm{L}_{3} \nsubseteq \mathrm{L}_{2}$. So 1 ) holds. From 1) it follows 2) and 3).

Lemma 2. 9. It holds that 1) $L_{2} \cap L_{31} \subsetneq L_{2}$ and 2) $L_{31} \subsetneq L_{32}$.

Proof. Consider the tpba $\left(P_{4}, I_{4}\right)$ in Figure 2.5. In $\left(P_{4}, I_{4}\right), I_{4} a=b$ and $I_{4} c=d$. The tpba $\left(P_{4}, I_{4}\right)$ is of type 31. Let $f(p)=b$ and $f(q)=c$. Then

$$
f\left(A_{2}\right)=(b \supset d) \supset I_{4}(b \supset d)=c \supset d=b \neq 1 .
$$

Hence $\mathrm{L}_{2} \nsubseteq \mathrm{L}_{31}$. Thus 1) and 2) hold.

Lemma 2. 10. It holds that $L_{32} \subsetneq L_{4}$.

Proof. The tpba $\left(P_{5}, I_{5}\right)$ in Figure 2.6 is clearly of type 32 . But $I_{5} a \cup I_{5}-I_{5} a=a \cup 0=a$. Hence, $I_{4} \nsubseteq \mathrm{L}_{32}$.

\section{§ 3. I Models}

In this section and $\S 5$, we introduce two kinds of Kripke models for IML's. They are obtained by extending either Kripke models for the intuitionistic logic or those for modal logics. So they are called to be $I$ models (intuitionistic-type Kripke models) and $M$ models (modaltype Kripke models), respectively.

A triple $(M, \leq, R)$ is an I frame, if

1) $\quad M$ is a nonempty set with a partial order $\leq$,

2) $R$ is a reflexive and transitive relation on $M$ such that $x \leq y$ implies $x R y$ for each $x, y \in M$.

A valuation $W$ on an $I$ frame $(M, \leq, R)$ is a mapping from the 
direct product of the set of formulas and the set $M$ to the set $\{t, f\}$, such that for any $a \in M$,

1) $W(p, a)=t$ and $a \leq b$ imply $W(p, b)=t$ for any propositional variable $p$,

2) $W(A \wedge B, a)=t$ if and only if $W(A, a)=t$ and $W(B, a)=t$.

3) $W(A \vee B, a)=t$ if and only if $W(A, a)=t$ or $W(B, a)=t$.

4) $W(A \supset B, a)=t$ if and only if for any $b$ such that $a \leq b$, $W(A, b)=f$ or $W(B, b)=t$.

5) $W(\neg A, a)=t$ if and only if for any $b$ such that $a \leq b, W(A, b)$ $=f$.

6) $W(\square A, a)=t$ if and only if for any $b$ such that $a R b, W(A, b)$ $=t$.

We remark that each value $W(A, a)$ is completely determined by values $W(p, b)$ for propositional variables $p$ and elements $b \in M$. A quadruple $(M, \leq, R, W)$ is an $I$ model, if $(M, \leq, R)$ is an $I$ frame and $W$ is a valuation on it. A formula $A$ is valid in an $I$ model $(M, \leq$, $R, W)$ if $W(A, a)=t$ for any $a$ in $M$. A formula $A$ is valid in an $I$ frame $(M, \leq, R)$ if it is valid in an $I$ model $(M, \leq, R, W)$ for any valuation $W$.

In the definition of an $I$ frame $(M, \leq, R)$, we may take a quasi-order (i.e. a reflexive, transitive relation) for $\leq$. More precisely, for any $I$ frame $(M, \leq, R)$ with a quasi-order $\leq$, there exists an $I$ frame $\left(M^{*}\right.$, $\leq^{*}, R^{*}$ ) with a partial order $\leq^{*}$ such that any formula is valid in $(M, \leq, R)$ if and only if it is valid in $\left(M^{*}, \leq^{*}, R^{*}\right)$. We can verify the following lemma.

Lemma 3. 1. Every formula provable in $L_{0}$ is valid in any $I$ model.

For any binary relation $R$, we write $x \sim_{R} y$ if $x R y$ and $y R x$ hold. Clearly, the relation $\sim_{R}$ is symmetric. We omit the subscript letter $R$ in the following definition. Define $I$ frames of type $J$ for $J=0,1,2,3$, $31,32,4$ as follows.

0) Any I frame is of type 0.

1) An I frame $(M, \leq, R)$ is of type 1 , when for each $x, y \in M$, 
if $x R y$ then there is an element $y^{\prime}$ in $M$ such that $x \leq y^{\prime}$ and $y R y^{\prime}$.

2) An I frame $(M, \leq, R)$ is of type 2, when for each $x, y \in M$, if $x R y$ then there is an element $y^{\prime}$ in $M$ such that $x \leq y^{\prime}$ and $y \sim y^{\prime}$.

3) An I frame $(M, \leq, R)$ is of type 3, when for each $x, y \in$ in $M$, if $x R y$ then there is an element $x^{\prime}$ in $M$ such that $x \sim x^{\prime}$ and $x^{\prime} \leq \nu$.

3j) An I frame is of type $3 j$ if it is both of type 3 and of type $j$ for $j=1,2$.

4) An I frame $(M, \leq, R)$ is of type 4 if $R$ is symmetric.

An $I$ model $(M, \leq, R, W)$ is of type $J$, if the $I$ frame $(M, \leq, R)$ is of type $J$. We remark that models for MIPC introduced in [3] are of type 2 , if we leave $\diamond$ out of consideration. When an $I$ frame $(M$, $\leq, R)$ validates the law of excluded middle, $x \leq y$ must imply $x=y$. In this case, we can see that the condition of an $I$ frame $(M, \leq, R)$ to be of type $J$, where $J \neq 0$, coincides with the condition that $R$ is symmetric. Now we show the completeness theorem for IML's with respect to $I$ models.

Theorem 3.2. A formula is provable in $L_{J}$ if and only if it is valid in any I model of type $J$, for $J=0,1,2,3,31,32,4$.

Proof. Only if part. By Lemma 3.1, we have only to prove that axioms of each IML added to $\mathrm{L}_{0}$ are valid in the corresponding $I$ models. Here we gire only a proof of the validity of $A_{2}$ in any $I$ model of type 2 . We first remark that for any formula $A$ and any $I$ model $(M, \leq, R, W)$, $x \sim x^{\prime}$ implies $W(\square A, x)=W\left(\square A, x^{\prime}\right)$. Now suppose that $A_{2}$ is not valid in an $I$ model $(M, \leq, R, W)$ of type 2 . Then there is an element $a$ in $M$ such that

(1) $\Pi^{r}(\square p \supset \square q, a)=t$,

(2) $W(\square(\square p \supset \square q), a)=f$.

By (2), $W(\square p \supset \square q, b)=f$ for some $b$ such that $a R b$. So, $W(\square p$, $c)=t$ and $W(\square q, c)=f$ for some $c$ such that $b \leq c$. Since $b \leq c$ implies $b R c, a R c$ holds by the transitivity of $R$. By the assumption that ( $M$, $\leq, R)$ is of type 2, there is an element $c^{\prime}$ such that $a \leq c^{\prime}$ and $c \sim c^{\prime}$. 
By (1), either $W\left(\square p, c^{\prime}\right)=f$ or $W\left(\square q, c^{\prime}\right)=t$. Using the above remark, it follows that $W(\square p, c)=f$ or $W(\square q, c)=t$. But this is a contradiction.

If part. We make use of a standard method. Let $\Phi$ be the set of formulas. Suppose that $\Gamma$ and $\Delta$ are subsets of $\Phi$. A pair $(\Gamma, \Delta)$ is said to be G-inconsistent, where $G$ is any one of sequent calculi $G_{J}$ 's, if there are formulas $A_{1}, \cdots, A_{m}$ in $\Gamma$ and $B_{1}, \cdots, B_{n}$ in $\Delta$ such that

$$
\mathrm{G} \vdash \mathrm{A}_{1}, \cdots, A_{m} \rightarrow B_{1}, \cdots, B_{n} .
$$

Otherwise, we say that $(\Gamma, \Delta)$ is G-consistent. A pair $(\Gamma, \Delta)$ is Gcomplete if it is G-consistent and $\Delta=\Gamma^{c}$, where $\Gamma^{c}$ denotes the complement of $\Gamma$ with respect to $\Phi$. Let $(\Gamma, \Delta)$ and $\left(\Gamma^{\prime}, \Delta^{\prime}\right)$ be G-consistent pairs. We say $\left(\Gamma^{\prime}, \Delta^{\prime}\right)$ is an extension of $(\Gamma, \Delta)$ if $\Gamma \subseteq \Gamma^{\prime}$ and $\Delta \subseteq \Delta^{\prime}$. By using Zorn's lemma, we can verify that for any G-consistent pair, there exists a G-complete extension of it. Now, let $M_{G}$ be the set $\{\Gamma ;(\Gamma, \Delta)$ is G-complete\}. We write $\Gamma_{\square}$ for the set $\{\square A ; \square A \in \Gamma\}$. Then, define a binary relation $R_{G}$ on $M_{G}$ by

$$
\Gamma_{1} R_{G} \Gamma_{2} \Leftrightarrow\left(\Gamma_{1}\right)_{\square} \subseteq\left(\Gamma_{2}\right)_{\square},
$$

where $\subseteq$ denotes the set inclusion. It is obvious that 1) $\Gamma_{1} \subseteq \Gamma_{2}$ implies $\Gamma_{1} R_{G} \Gamma_{2}$ and 2) $R_{G}$ is a reflexive and transitive relation. Thus, ( $M_{G}, \subseteq$, $\left.R_{G}\right)$ is an $I$ frame, for any sequent calculus $G$ under consideration.

A valuation $W_{G}$ on $\left(M_{G}, \subseteq, R_{G}\right)$ is defined by

$$
W_{G}(p, \Gamma)=t \Leftrightarrow p \in \Gamma,
$$

for any propositional variable $p$. Then we can show by induction that for any formula $A$,

$$
W_{G}(A, \Gamma)=t \Leftrightarrow A \in \Gamma .
$$

Here we give a proof of this only for the case where $A$ is $\square B$. Suppose that $\square B \in \Gamma$. Let $\Gamma^{\prime}$ be any element of $M_{G}$ such that $\Gamma R_{G} \Gamma^{\prime}$. Then $\square B \in \Gamma^{\prime}$. Since $\mathrm{G}_{0} \vdash \square B \rightarrow B$ and $\left(\Gamma^{\prime}, \Gamma^{\prime c}\right)$ is G-complete, $B$ must be in $\Gamma^{\prime}$. So, by the induction hypothesis, $W_{G}\left(B, \Gamma^{\prime}\right)=t$. Hence $W_{G}(\square B, \Gamma)$ $=t$. Suppose next that $\square B \notin \Gamma$. Then $(\Gamma,\{\square B\})$ is G-consistent, since $\Gamma$ is in $M_{G}$. Now we show that $\left(\Gamma_{\square},\{B\}\right)$ is G-consistent. Suppose otherwise. Then there are formulas $\square A_{1}, \cdots, \square A_{m}$ in $\Gamma$ such that 
$G \vdash \square A_{1}, \cdots, \square A_{m} \rightarrow B$. Since $\mathrm{G}$ is equal to or stronger than $\mathrm{G}_{0}, \mathrm{G} \vdash \square \mathrm{A}_{1}$, $\cdots, \square A_{m} \rightarrow \square B$. But this contradicts the G-consistency of $(\Gamma,\{\square B\})$. Hence, $\left(\Gamma_{\square},\{B\}\right)$ is G-consistent. Let $\left(\Pi, \Pi^{c}\right)$ be a G-complete extension of $\left(\Gamma_{\square},\{B\}\right)$. Then $\Gamma R_{G} I I$ holds by the definition. Moreover, $B \notin \Pi$. So, by the induction hypothesis, $W_{G}(B, \Pi)=f$. Thus $W_{G}(\square B, \Gamma)=f$.

Now, we show the completeness of $\mathrm{L}_{0}$. Let $A$ be any formula not provable in $\mathrm{L}_{0}$. Then, by Theorem $2.1(\varnothing,\{A\})$ is $\mathrm{G}_{0}$-consistent. There is an $\mathrm{G}_{0}$-complete extension $\left(\Gamma, \Gamma^{c}\right)$ of $(\varnothing,\{A\})$. Since $A \notin I$, $W_{G_{0}}(A, \Gamma)=f$ in $\left(M_{G_{0}}, \subseteq, R_{G_{0}}\right)$. Thus there is an $I$ model of type 0 in which $A$ is not valid.

In order to prove the completeness of other IML's, it is sufficient to show that the $I$ frame $\left(M_{G_{J}}, \subseteq, R_{G_{J}}\right)$ thus constructed is of type $J$.

Consider the case where $J=2$. We will show that if $\Gamma R_{G_{2}} \Gamma^{\prime}$ then there is $I$ in $M_{G_{2}}$ such that $\Gamma \subseteq \Pi$ and $\Gamma_{\square}^{\prime}=\Pi_{\square}$.

Assume that $\Gamma R_{G_{2}} \Gamma^{\prime}$. We first show that $\left(\Gamma \cup \Gamma_{\square}^{\prime},\left(\Gamma^{\prime c}\right)_{\square}\right)$ is $\mathrm{G}_{2^{-}}$ consistent. Suppose otherwise. Then there exist formulas $A_{1}, \cdots, A_{k}$ in $\Gamma, \square B_{1}, \cdots, \square B_{m}$ in $\Gamma^{\prime}$ and $\square C_{1}, \cdots, \square C_{n}$ in $\Gamma^{\prime c}$ such that

$$
G_{2} \vdash A_{1}, \cdots, A_{k}, \square B_{1}, \cdots, \square B_{m} \rightarrow \square C_{1}, \cdots, \square C_{n} .
$$

Then,

$$
\mathrm{G}_{2} \vdash A_{1}, \cdots, A_{k} \rightarrow \bigwedge_{i=1}^{m} \square B_{i} \supset \bigvee_{j=1}^{n} \square C_{j}
$$

By using $(\rightarrow \square 2)$ and cut,

$$
\mathrm{G}_{2} \vdash A_{1}, \cdots, A_{k} \rightarrow \square\left(\bigwedge_{i=1}^{n} \square B_{i} \supset \bigvee_{j=1}^{n} \square C_{j}\right)
$$

From the assumption that each $A_{i}$ is in $\Gamma$, it follows that $\square\left(\bigwedge_{i=1}^{m} \square B_{i}\right.$ $\left.\supset \bigvee_{j=1}^{n} \square C_{j}\right) \in \Gamma$. Since $\Gamma_{\square} \subseteq \Gamma^{\prime}{ }_{\square}$ by the assumption, $\square\left(\bigwedge_{i=1}^{m} \square B_{i} \supset \bigvee_{j=1}^{n} \square C_{j}\right)$ $\in \Gamma^{\prime}$. Thus $\bigwedge_{i=1}^{m} \square B_{i} \supset \bigvee_{j=1}^{n} \square C_{j} \in \Gamma^{\prime}$. Since each $\square B_{i}$ is in $\Gamma^{\prime}, \bigwedge_{i=1}^{m} \square B_{i}$ $\in \Gamma^{\prime}$ and hence $\bigvee_{j=1}^{n} \square C_{j} \in \Gamma^{\prime}$. So some $\square C_{j}$ must be in $\Gamma^{\prime}$. But this is a contradiction. Thus $\left(\Gamma \cup \Gamma_{\square}^{\prime},\left(\Gamma^{\prime c}\right)_{\square}\right)$ is $\mathrm{G}_{2}$-consistent. Now, let $\left(\Pi, \Pi^{c}\right)$ be a $\mathrm{G}_{2}$-complete extension of $\left(\Gamma \cup \Gamma_{\square}^{\prime},\left(\Gamma^{\prime c}\right)_{\square}\right)$. It is clear that $\Gamma \subseteq \Pi$ and $\Gamma^{\prime}{ }_{\square} \subseteq \Pi_{\square}$. Let $\square D \notin \Gamma^{\prime}{ }_{\square}$. Then $\square D \in\left(\Gamma^{\prime c}\right)_{\square} \subseteq \Pi^{c}$. Thus, $\square D \notin \Pi_{\square}$. So, $\Pi_{\square} \subseteq \Gamma_{\square}^{\prime}$ and hence $\Gamma_{\square}^{\prime}=\Pi_{\square}$. 
The completeness of $\mathrm{L}_{1}$ can be proved similarly as above. Next, consider the case $J=3$. We will show that if $\Gamma R_{G_{3}} \Gamma^{\prime}$ then there is $\Pi$ in $M_{G_{3}}$ such that $\Gamma_{\square}=\Pi_{\square}$ and $\Pi \subseteq \Gamma^{\prime}$. Assume that $\Gamma R_{G_{3}} \Gamma^{\prime}$. We prove that $\left(\Gamma_{\square},\left(\Gamma^{c}\right)_{\square} \cup \Gamma^{\prime c}\right)$ is $\mathrm{G}_{3}$-consistent. Suppose otherwise. Then there are formulas $\square A_{1}, \cdots, \square A_{k}$ in $\Gamma, \square B_{1}, \cdots, \square B_{m}$ in $\Gamma^{c}$ and $C_{1}, \cdots, C_{n}$ in $\Gamma^{\prime c}$ such that

$$
\mathrm{G}_{3} \vdash \square A_{1}, \cdots, \square A_{k} \rightarrow \square B_{1}, \cdots, \square B_{m}, C_{1}, \cdots, C_{n} .
$$

Hence,

$$
\mathrm{G}_{3} \vdash \square A_{1}, \cdots, \square A_{k} \rightarrow \square B_{1}, \cdots, \square B_{m}, \bigvee_{i=1}^{n} C_{i}
$$

By the rule $(\rightarrow \square+0)$,

$$
\mathrm{G}_{3} \vdash \square A_{1}, \cdots, \square A_{k} \rightarrow \square B_{1}, \cdots, \square B_{m}, \square \bigvee_{i=1}^{n} C_{i}
$$

Suppose $\square \bigvee_{i=1}^{n} C_{i} \in \Gamma$. Since $\Gamma R_{G_{3}} \Gamma^{\prime}, \square \bigvee_{i=1}^{n} C_{i} \in \Gamma^{\prime}$ and hence $\bigvee_{i=1}^{n} C_{i} \in \Gamma^{\prime}$. So, some $C_{i}$ is in $\Gamma^{\prime}$. But this is a contradiction. Hence $\square \bigvee_{i=1}^{n} C_{i} \in \Gamma^{c}$. Then it follows from (3) that $\left(\Gamma, \Gamma^{c}\right)$ is $\mathrm{G}_{3}$-inconsistent, contrary to our assumption. Thus $\left(\Gamma_{\square},\left(\Gamma^{c}\right)_{\square} \cup \Gamma^{\prime c}\right)$ is $\mathrm{G}_{3}$-consistent. Let $\left(\Pi, \Pi^{c}\right)$ be a $\mathrm{G}_{3}$-complete extension of $\left(\Gamma_{\square},\left(\Gamma^{c}\right)_{\square} \cup \Gamma^{\prime c}\right)$. We have that $\Pi \subseteq \Gamma^{\prime}$, since $\Gamma^{\prime c} \subseteq \Pi^{c}$. Let $\square D \notin \Gamma_{\square}$. Then $\square D \in\left(\Gamma^{c}\right)_{\square} \subseteq \Pi^{c}$. Thus $\Pi_{\square} \subseteq \Gamma_{\square}$. Clearly, $\Gamma_{\square} \subseteq \Pi_{\square}$. Hence $\Gamma_{\square}=\Pi_{\square}$. So we have the completeness of $\mathrm{L}_{3}$. Combining this with the proof of the completeness of $L_{1}$ and $L_{2}$, we get also the completeness of $\mathrm{L}_{31}$ and $\mathrm{L}_{32}$.

Finally, consider the case $J=4$. We must show that $R_{G_{4}}$ is symmetric. Suppose that $\Gamma R_{G_{4}} \Gamma^{\prime}$ and that there is a formula $\square A$ such that $\square A \in \Gamma^{\prime}-\Gamma$. Since $\square A \vee \square \neg \square A$ is provable in $\mathrm{G}_{4}$, it must be in $\Gamma$. So, either $\square A \in \Gamma$ or $\square \neg \square A \in \Gamma$. But since $\square A \notin \Gamma, \square \neg \square A \in \Gamma$. By the assumption that $\Gamma R_{G_{4}} \Gamma^{\prime}, \square \neg \square A \in \Gamma^{\prime}$. On the other hand, $\square A$ $\in \Gamma^{\prime}$. But, it is clear that $\mathrm{G}_{4} \vdash \square A, \square \neg \square A \rightarrow$. This contradicts the $\mathrm{G}_{4}$-consistency of $\left(\Gamma^{\prime}, \Gamma^{\prime c}\right)$. Thus, $\Gamma_{\square}=\Gamma^{\prime}$. This means that $R_{G_{4}}$ is symmetric. This completes the proof of the theorem.

Similarly as Theorem 3.2, we can obtain the completeness theorem for other IML's. For example, consider an IML $\widetilde{\mathrm{L}}$ obtained from the 
intuitionistic logic $\mathrm{H}$ by adding the axioms (1) and (3) in $\S 2$. Then $\widetilde{\mathrm{L}}$ is an IML of T-type. An $\widetilde{I}$ frame $(M, \leq, R)$ is a triple of a set $\mathrm{M}$, a partial order $\leq$ on $M$ and a reflexive relation $R$ on $M$. Then we can show that $\widetilde{\mathrm{L}}$ is complete with respect to $\widetilde{I}$ frames.

Using Theorem 3.2, we show that sequent calculi $K_{0}$ and $G_{0}$ are cut-free. We employ the method due to Fitting [5]. We use the terminology of [5]. A $\mathrm{G}_{0}$-consistency property (or a $\mathrm{K}_{0}$-consistency property) is obtained from the propositional part of a Beth intuitionistic consistency property (or a Gentzen intuitionistic consistency property, respectively) by adding the following conditions for $\square$;

i) If $T \square A \in S$ then $S \cup\{T A\} \in \mathscr{C}$,

ii) if $F \square A \in S$ then $S_{T \square} \cup\{F A\} \in \mathscr{C}$,

where $S_{T \square}=\{T \square B ; T \square B \in S\}$. Similarly as [5], we can show that every $\mathrm{G}_{0}$-consistency property can be extended to a $\mathrm{K}_{0}$-consistency property。

Let $S \in \mathscr{C}$, where $\mathscr{C}$ is a $\mathrm{K}_{0}$-consistency property. We say $S$ is T-saturated if

1) if $p$ is a propositional variable, not both $T p \in S$ and $F p \in S$,

2) if $T(A \wedge B) \in S$ then $T A \in S$ and $T B \in S$,

$3)$ if $T(A \vee B) \in S$ then $T A \in S$ or $T B \in S$,

4) if $T(A \supset B) \in S$ and $S \cup\{T B\} \in \mathscr{C}$ then $T B \in S$,

5) if $T \square A \in S$ then $T A \in S$.

Then we can prove the key lemma similarly as [5]. Let $S_{0}$ be a set of signed formulas, belonging to a $\mathrm{K}_{0}$-consistency property $\mathscr{C}$. By the key lemma, we can assume that $\mathscr{C}$ is closed under chain unions. Let $M$ be the set of all $T$-saturated elements in $\mathscr{C}$. Then there is some $S$ in $M$ such that $S$ is an extension of $S_{0}$. For each $S, S^{\prime} \in M$, define $S \leq S^{\prime}$ if $S_{T} \subseteq S_{T}^{\prime}$, where $S_{T}=\{T B ; T B \in S\}$. Clearly $\leq$ is a quasi-order. For each $S, S^{\prime} \in M$, define $S R S^{\prime}$ if $S_{T_{\square}} \subseteq S_{T_{\square}}^{\prime}$. If $S \leq S^{\prime}$ then $S_{T_{\square}} \subseteq S_{T}$ $\subseteq S^{\prime}$. Hence $S R S^{\prime}$. Thus, $(M, \leq, R)$ is an $I$ frame of type 0 . (See the remark just above Lemma 3.1.) Now, define a valuation $W$ on $(M, \leq, R)$ by

$$
W(p, s)=t \Leftrightarrow T p \in S
$$

for any propositional variable $p$ and any $S \in M$. Then we can show that for any $S \in M$ and any formula $A$ 
1) $T A \in S$ implies $W(A, S)=t$,

2) $F A \in S$ implies $W(A, S)=f$.

Hence $S_{0}$ is satisfiable. Thus we get the model existence theorem for $\mathrm{K}_{0}$ and $\mathrm{G}_{0}$. As a corollary, we have the completeness of $\mathrm{K}_{0}$ (or $\mathrm{G}_{0}$ ) without cut, with respect to $I$ frames of type 0 . Combining this with Theorem 2.1 and 3.2, we have the following theorem.

Theorem 3. 3. Any sequent provable in the sequent calculus $K_{0}$ (or $G_{0}$ ) is provable without cut in $K_{0}$ (or $G_{0}$, respectively).

We remark that A. Yamamoto proved this theorem syntactically. Next, we show a connection between tpba's and $I$ frames. Let $(M, \leq, R)$ be an $I$ frame of type $J$. A subset $S$ of $M$ is said to be closed if $a \in S$ and $a \leq b$ implies $b \in S$. Let $P_{M}$ be the set of all closed subsets of $M$. Then $P_{M}$ is a pseudo-Boolean algebra with respect to set operations. Note that for every $S, T \in P_{M}$,

$$
S \supset T=\{a ; \text { for any } b \text { such that } a \leq b, b \in(M-S) \cup T\}
$$

and

$$
\sim S=\{a ; \text { for any } b \text { such that } a \leq b, b \notin S\} .
$$

Define an operation $I$ on $P_{M}$ by

$$
I S=\{a ; \text { for any } b \text { such that } a R b, b \in S\} .
$$

It is easy to see that $\left(P_{M}, I\right)$ is a tpba. We can show also that $\left(P_{M}, I\right)$ is of type $J$. This can be proved quite similarly as only if part of Theorem 3.2. Now we get the following theorem.

Theorem 3. 4. Suppose that $(M, \leq, R)$ is an I frame of type $J$. Let $P_{M}$ be the pseudo-Boolean algebra consisting of the set of all closed subsets of $M$ and $I$ be an operation defined by (4). Then, $\left(P_{M}, I\right)$ is a tpba of type $J$ such that for any formula $A, A$ is valid in $(M, \leq, R)$ if and only if $A$ is valid in $\left(P_{M}, I\right)$.

Conversely, consider a construction of an $I$ frame corresponding to a given tpba. As in the case of pseudo-Boolean algebras, we can only 
prove a weaker result. Suppose that $(P, I)$ is a tpba of type $J$. Let $M_{P}$ be the set of all prime filters of $P$. Clearly, $M_{P}$ is partially ordered by the set inclusion $\subseteq$. For any $F$ in $M_{P}, F_{I}$ denotes the set $\{I a$; $I a \in F\}$. Now, define a binary relation $R$ on $M_{P}$ by

$$
F R G \Leftrightarrow F_{I} \subseteq G_{I} .
$$

Then we can show that $\left(M_{P}, \subseteq, R\right)$ is an $I$ frame of type $J$. Also, this can be proved quite similarly as if part of Theorem 3.2. We have the following theorem (cf. [13]).

Theorem 3.5. Suppose that $(P, I)$ is any tpba of type J. Let $M_{P}$ be the sci of all prime filters of $P$. Then the I frame $\left(M_{P}, \subseteq, R\right)$, where $R$ is defined by (5), is of type $J$. For any assignment $f$ of $(P, I)$, let $W$ be a valuation on $\left(M_{P}, \subseteq, R\right)$ such that $W(p, F)=t$ $\Leftrightarrow f(p) \in F$, for any propositional variable $p$. Then, for any formula $A, f(A)=1$ if and only if $A$ is valid in the I model $\left(M_{P}, \subseteq, R, W\right)$. Furthermore, when $P$ is finite, it holds that a formula is valid in $(P, I)$ if and only if it is valid in $\left(M_{P}, \subseteq, R\right)$.

We notice here that each $I$ frame $(M, \leq, R)$ can be considered as a Kripke frame of a bimodal logic. That is, $R$ gives an interpretation for a stronger necessity and $\leq$ for a weaker necessity. Thus, we can define bimodal logics corresponding to each type of $I$ frames. For example, as proved in [8], the bimodal logic S4-S4 is complete with respect to $I$ frames (of type 0 ). In other words, each IML can be embedded into some bimodal logic by a standard translation.

\section{§4. The Finite Model Property for $\mathrm{HML}$ 's}

We prove in this section that every IML under consideration except $\mathrm{L}_{1}$ and $\mathrm{L}_{31}$ has the finite model property. By the finite model property for an IML $\mathrm{L}_{J}$, we mean that for any formula $A$ not provable in $\mathrm{L}_{J}$, there is a finite tpba of type $J$ in which $A$ is not valid. By Theorems 3. 4 and 3.5, this is equivalent to the condition that for any formula $A$ not provable in $\mathrm{L}_{J}$, there is a finite $I$ frame of type $J$ in which $A$ is not valid. The finite model property for $\mathrm{L}_{0}$ is proved by Bull [2]. 
We don't know whether $L_{1}$ and $L_{31}$ have the finite model property.

By modifying the proof of Theorem 3.2 , we can prove the finite model property for $\mathrm{L}_{0}$. Let $A$ be any formula not provable in $\mathrm{L}_{0}$. Our proof proceeds in the similar manner as the if part of Theorem 3.2. This time, we take the set of all subformulas of $A$ for $\Phi$, instead of the set of all formulas. Using this $\Phi$, we define an $I$ model $\left(M_{G_{0}}, \subseteq, R_{G_{0}}\right.$, $W_{G_{0}}$ ) in the same way as before. The $I$ frame thus obtained is finite, since $\Phi$ is finite. Moreover we can show that this $I$ model is of type 0 and that $A$ is not valid in it. The method of proving the finite model property by taking a finite set $\Phi$ is developed by Schütte [21] for the intuitionistic propositional logic and by Sato [20] for some classical modal logics.

Theorem 4. 1. (Bull) $L_{0}$ has the finite model property and hence is deciable.

To show the finite model property for $\mathrm{L}_{4}$, we can use the filtration method. But we give here a more direct proof suggested by Sato.

Theorem 4.2. $L_{4}$ has the finite model property and hence is decidable.

Proof. Suppose that $A$ is any formula not provable in $\mathrm{L}_{4}$ and $\Phi$ is the set of all subformulas of $A$. Let $M$ be the set $\{\Gamma ;(\Gamma, \Delta)$ is $\mathrm{G}_{4}$-complete in $\left.\Phi\right\}$. Clearly, $M$ is finite. Define two binary relations $R$ and $\leq$ on $M$ by

$$
\Gamma R \Gamma^{\prime} \Leftrightarrow \Gamma_{\square}=\Gamma_{\square}^{\prime},
$$

and

$$
\Gamma \leq \Gamma^{\prime} \Leftrightarrow \Gamma R \Gamma^{\prime} \text { and } \Gamma \subseteq \Gamma^{\prime} .
$$

Then it is clear that $\Gamma \leq \Gamma^{\prime}$ implies $\Gamma R \Gamma^{\prime}$ and that $(M, \leq, R)$ is an $I$ frame of type 4. A valuation $W$ on $(M, \leq, R)$ is defined by

$$
W(p, \Gamma)=t \Leftrightarrow p \in \Gamma .
$$

Then, we can prove that for any formula $B$ in $\Phi$, 


$$
W(B, \Gamma)=t \Leftrightarrow B \in \Gamma .
$$

To show this, it suffices to prove that

1) for any formula $B \supset C$ in $\Phi$,

$$
\begin{gathered}
B \supset C \in \Gamma \Leftrightarrow \text { for any } \Gamma^{\prime} \in M \text { such that } \Gamma \leq \Gamma^{\prime} \\
\text { either } B \notin \Gamma^{\prime} \text { or } C \in \Gamma^{\prime},
\end{gathered}
$$

2) for any formula $\neg B$ in $\Phi$,

$\neg B \in \Gamma \Leftrightarrow$ for any $\Gamma^{\prime} \in M$ such that $\Gamma \leq \Gamma^{\prime}$,

$$
B \notin \Gamma^{\prime} \text {, }
$$

3) for any formula $\square B$ in $\Phi$,

$\square B \in \Gamma \Leftrightarrow$ for any $\Gamma^{\prime} \in M$ such that $\Gamma R \Gamma^{\prime}$,

$$
B \in \Gamma^{\prime} \text {. }
$$

Suppose that $B \supset C \in \Gamma$ and $\Gamma \leq \Gamma^{\prime}$. Then $B \supset C \in \Gamma^{\prime}$, since $\Gamma \leq \Gamma^{\prime}$ implies $\Gamma \subseteq \Gamma^{\prime}$. Since $\mathrm{G}_{4} \vdash B, B \supset C \rightarrow C, B \in \Gamma^{\prime}$ implies $C \in \Gamma^{\prime}$. That is, $B \notin \Gamma^{\prime}$ or $C \in \Gamma^{\prime}$. Conversely, suppose that $B \supset C \notin \Gamma$. We show that $\left(\Gamma \cup\{B\},\left(\Gamma^{c}\right)_{\square} \cup\{C\}\right)$ is $\mathrm{G}_{4}$-consistent. Suppose otherwise. Then there exist $A_{1}, \cdots, A_{m}$ in $\Gamma$ and $\square D_{1}, \cdots, \square D_{n}$ in $\left(\Gamma^{c}\right)_{\square}$ such that

$$
\mathrm{G}_{4} \vdash B, A_{1}, \cdots, A_{m} \rightarrow \square D_{1}, \cdots, \square D_{n}, C .
$$

Then by using $\left(\rightarrow \supset^{*}\right)$,

$$
\mathrm{G}_{4} \vdash A_{1}, \cdots, A_{m} \rightarrow \square D_{1}, \cdots, \square D_{n}, B \supset C .
$$

But this contradicts to the $\mathrm{G}_{4}$-consistency of $\left(\Gamma, \Gamma^{c}\right)$. Thus, $(\Gamma \cup\{B\}$, $\left.\left(\Gamma^{c}\right)_{\square} \cup\{C\}\right)$ is $\mathrm{G}_{4}$-consistent. Then for some $\Gamma^{\prime} \in M, \Gamma \cup\{B\} \subseteq \Gamma^{\prime}$ and $\left(\Gamma^{c}\right)_{\square} \cup\{C\} \subseteq\left(\Gamma^{\prime}\right)^{c}$. Clearly, $B \in \Gamma^{\prime}$ and $C \notin \Gamma^{\prime}$. Moreover, $\Gamma_{\square}=\Gamma^{\prime}$ 口 and $\Gamma \subseteq \Gamma^{\prime}$. Thus, $\Gamma \leq \Gamma^{\prime}$. 2) and 3) can be shown similarly. Now, since $A \notin \Gamma$ for some $\Gamma \in M, A$ is not valid in $(M, \leq, R, W)$.

For other IML's, the method used in Theorems 4.1 and 4.2 does not work well. We show the finite model property for other IML's by using tpba's. Our method is a generalization of the one by Bull [2].

Theorem 4. 3. $L_{3}$ has the finite model property and hence is decidable.

Proof. Let $A$ be a formula not provable in $L_{3}$. By Theorem 2.2, 
there is a tpba $(P, I)$ of type 3 and an assignment $f$ of $(P, I)$ such that $f(A) \neq 1$. $\cap, \cup$ and $\supset$ denote join, meet and relative pseudo-complement in $P$. The greatest element and the least element in $P$ are denoted by 1 and 0 , respectively. We enumerate the value of each subformula of $A$ by $f$ as $a_{1}, \cdots, a_{m}$. For each nonempty subset $U$ of $\left\{a_{1}, \cdots, a_{m}\right\}$, define an element $U^{*}$ by $U^{*}=b_{1} \cup \cdots \cup b_{k}$, if $U=\left\{b_{1}, \cdots, b_{k}\right\}$. Now, let $S=\left\{0,1, a_{1}, \cdots, a_{m}\right\} \cup\left\{I U^{*} ; U\right.$ is a nonempty subset of $\left.\left\{a_{1}, \cdots, a_{m}\right\}\right\}$. Let $P_{0}$ be the sublattice of $P$ generated by the set $S$. Since $S$ is finite, $P_{0}$ is also finite. For each $x, y \in P_{0}$, define $x \supset y$ by

$$
x \supset y=\bigcup_{z}\left\{z ; z \in P_{0} \text { and } z \leq x \supset y\right\} \text {. }
$$

Then, $x \supset y$ is also in $P_{0}$ and is the relative pseudo-complement of $x$ in $P_{0}$ with respect to $y$. Remark that if $x \supset y \in P_{0}$ for $x, y \in P_{0}$ then $x \supset y$ $=x \supset y$. Furthermore, $P_{0}$ with the operation $\cap, \cup, \supset$ and the least element 0 constitutes a pseudo-Boolean algebra. We will show that for any $a \in P_{0} I a \in P_{0}$. Suppose that $a \in P_{0}$. Since $P$ is a distributive lattice, so is $P_{0}$. Hence $a$ can be represented in the conjunctive form. So, we let $a=\bigcap_{i=1}^{n}\left(\bigcup_{j=1}^{k_{i}} b_{i j}\right)$, where each $b_{i j} \in S$. Then, $I a=\bigcap_{i=1}^{n} I\left(\bigcup_{j=1}^{k_{i}} b_{i j}\right)$. It is sufficient to show that $I\left(\bigcup_{j=1}^{k_{i}} b_{i j}\right) \in P_{0}$. Without a loss of generality, we may suppose that among $b_{i 1}, \cdots, b_{i k_{i}}$, elements $b_{i 1}, \cdots, b_{i n}$ are open and others are not. Then,

$$
\begin{aligned}
I\left(\bigcup_{j=1}^{k_{i}} b_{i j}\right) & =I\left(\bigcup_{j=1}^{n} I b_{i j} \cup \bigcup_{j=n+1}^{k_{i}} b_{i j}\right) \\
& =\bigcup_{j=1}^{h} I b_{i j} \cup I\left(\bigcup_{j=h+1}^{k_{i}} b_{i j}\right)
\end{aligned}
$$

(by v) in $\S 2$, since $(P, I)$ is of type 3 )

$$
=\bigcup_{j=1}^{h} b_{i j} \cup I\left(\bigcup_{j=h+1}^{k_{i}} b_{i j}\right) \text {. }
$$

It is obvious that an element in $S$ which is not open is among $\underset{k_{i}}{a_{1}, \cdots, a_{m}}$. So, $I\left(\bigcup_{j=n+1}^{k_{i}} b_{i j}\right) \in S$ by the definition of $S$. Since $b_{i 1}, \cdots, b_{i n}$ and $I\left(\bigcup_{j=n+1}^{k_{i}} b_{i j}\right)$ are in $S, I\left(\bigcup_{j=1}^{k_{i}} b_{i j}\right) \in P_{0}$. Let $I_{0}$ be the restriction of $I$ to $P_{0}$. It is easy to see that $\left(P_{0}, I_{0}\right)$ is a tpba of type 3 , since $(P, I)$ is of type 3 . Now define an assignment $g$ of $\left(P_{0}, I_{0}\right)$ by $g(p)=f(p)$ if $p$ is any propositional variable appearing in the formula $A$. Then we can show that for any 
subformula $B$ of $A, g(B)=f(B)$. In particular, $g(A)=f(A) \neq 1$. Thus, $A$ is not valid in $\left(P_{0}, I_{0}\right)$.

To show the finite model property for $\mathrm{L}_{2}$ and $\mathrm{L}_{32}$, we introduce two IML's containing $\diamond$ as primitive, which are extensions of $\mathrm{L}_{2}$ and $\mathrm{L}_{32}$, respectively. Consider the following axioms concerning $\diamond$.

(1) $p \supset \diamond p$,

(2) $\diamond \diamond p \supset \diamond p$

(3) $\square(p \supset q) \supset(\diamond p \supset \diamond q)$,

(4) $\diamond(p \vee q) \supset(\diamond p \vee \diamond q)$,

(5) $\diamond p \supset \square \diamond p$,

(6) $\diamond \square p \supset \square p$.

Let $\mathrm{L}_{2^{*}}$ and $\mathrm{L}_{32^{*}}$ be IML's obtained from $\mathrm{L}_{2}$ and $\mathrm{L}_{32}$, respectively, by adding the above axioms. It can be easily verified that $L_{2^{*}}$ is equivalent to MIPC in [18].

Lemma 4. 4. $L_{2^{*}}$ and $L_{32^{*}}$ are conservative extensions of $L_{2}$ and $L_{32}$, respectively. More precisely, any formula not containing $\diamond$ is provable in $L_{2}\left(\right.$ or $L_{32}$ ) if it is provable in $L_{2^{k}}$ (or $L_{32^{*}}$ ).

Proof. Let $(M, \leq, R, W)$ be an $I$ model of type 2 . We extend $W$ so that $W(A, a)$ is defined also for any formula $A$ containing $\diamond$. Let us define $W(\diamond A, a)=t$ if and only if for some $b$ such that $a \sim b$, $W^{\prime}(A, b)=t$. We first confirm that for each $a, b \in M$, if $W(\diamond A, a)=t$ and $a \leq b$ then $W^{\top}(\diamond A, b)=t . \quad B y(D A, a)=t$, there is some $a^{\prime}$ such that $a \sim a^{\prime}$ and $W\left(A, a^{\prime}\right)=\ell$. Since $a \sim a^{\prime}$ implies $a^{\prime} R a$ and $a \leq b$ implies $a R b, a^{\prime} R b$ holds by the transitivity of $R$. As $(M, \leq, R)$ is of type 2, there exists an element $b^{\prime}$ such that $a^{\prime} \leq b^{\prime}$ and $b \sim b^{\prime}$. So, from $a^{\prime} \leq b^{\prime}$ and the induction hypothesis it follows that $W\left(A, b^{\prime}\right)=t$. Hence $W(\diamond A, b)=t$.

We say that the $I$ model thus obtained is the $\diamond$-extension of ( $M$, $\leq, R, W)$. It is easy to see that each axiom of $\mathrm{L}_{2^{\prime}}$ (or $\mathrm{L}_{32^{*}}$ ) is valid in the $\diamond$-extension of any $I$ model of type 2 (or type 32). Hence, 
any formula provable in $\mathrm{L}_{2^{*}}$ (or $\mathrm{L}_{32^{*}}$ ) is valid in the $\diamond$-extension of any $I$ model of type 2 (or type 32 ). Now, suppose that a formula $A$ not containing $\diamond$ is not provable in $\mathrm{L}_{2}$ (or $\mathrm{L}_{32}$ ). Then by Theorem 3.2, there is an $I$ model of type 2 (or type 32 ) in which $A$ is not valid. Clearly, $A$ is not valid also in its $\diamond$-extension. Thus, $A$ is not provable in $\mathrm{L}_{2^{\star}}$ (or $\mathrm{L}_{3 \varepsilon^{*}}$ ).

A triple $(P, I, C)$ is a bi-topological pseudo-Boolean algebra (abbreviated as $b i$-tpba), if

1) $(P, I)$ is a tpba,

2) $C$ is a unary operation on $P$ such that

i) $\quad C(a \cup b)=C a \cup C b$,

ii) $a \leq C a$,

iii) $C a=C C a$,

iv) $I(a \supset b) \leq C a \supset C b$,

v) $C I a=I a$,

vi) $\mathrm{ICa}=\mathrm{Ca}$.

We say that an element $a$ in $P$ is open (or closed) in a bi-tpba $(P, I, C)$ if $I a=a$ (or $C a=a$, respectively). By v) and vi), an element is open if and only if it is closed. So, we say that an element is clopen, if it is either open or closed. A bi-tpba $(P, I, C)$ is said to of type 2, if the set of all clopen elements in $(P, I, C)$ constitutes a sub- (pseudoBoolean) algebra of $P$. A bi-tpba is said to be of type 32, if it is of type 2 and $I(I a \cup b) \leq I a \cup I b$ holds in it. It is clear that if $(P, I, C)$ is of type 2 (or of type 32 ) then $(P, I$ ) is also of type 2 (or type 32 ). Note that the condition iv) in the above definition is redundant, when a bi-tpba under consideration is of type 2 . An assignment $f$ of a bi-tpba $(P, I, C)$ is an assignment of the tpba $(P, I)$ with $f(\diamond A)=C f(A)$ for any formula $A$. Similarly as Theorem 2.2, we have the following theorem.

Theorem 4.5. A formula is provable in $L_{2^{*}}\left(\right.$ or $\left.L_{32}\right)$ if and only if it is valid in any bi-tpba of type 2 (or of type 32, re- 
spectively).

We show here that in any bi-tpba $(P, I, C)$,

$$
C a \cap C b=C(C a \cap b)
$$

holds for every $a, b \in P$. By the condition i), $d \leq e$ implies $C d \leq C e$ for every $d, e$. Using this, it follows that $C(C a \cap b) \leq C a \cap C b$. On the other hand, since $C a \cap b \leq C(C a \cap b), C a \leq(b \supset C(C a \cap b))$. Then

$$
\begin{aligned}
C a=I C a & \leq I(b \supset C(C a \cap b)) \\
& \leq C b \supset C C(C a \cap b) \\
& =C b \supset C(C a \cap b) .
\end{aligned}
$$

Thus, $C a \cap C b \leq C(C a \cap b)$. Now we show the finite model property for $\mathrm{L}_{32^{*}}$.

Theorem 4. 6. $L_{32}$ has the finite model property and hence is decidable.

Proof. Our proof proceeds similarly as the proof of Theorem 4. 3. Let $A$ be a formula not provable in $\mathrm{L}_{32^{*}}$. Then there is a bi-tpba ( $P$, $I, C)$ of type 32 and its assignment $f$ such that $f(A) \neq 1$. Let $a_{1}, \cdots, a_{m}$ be values of each subformula of $A$ by $f$. For any nonempty subset $U$ of $\left\{a_{1}, \cdots, a_{m}\right\}$, define $U_{*}=b_{1} \cap \cdots \cap b_{k}$ if $U=\left\{b_{1}, \cdots, b_{k}\right\}$. Let $S=\{0,1$, $\left.a_{1}, \cdots, a_{m}\right\} \cup\left\{I U^{*} ; U\right.$ is a nonempty subset of $\left.\left\{a_{1}, \cdots, a_{m}\right\}\right\} \cup\left\{C U_{*} ; U\right.$ is a nonempty subset of $\left.\left\{a_{1}, \cdots, a_{m}\right\}\right\}$. Let $P_{0}$ be the sublattice of $P$ generated by $S$. Then $P_{0}$ becomes a finite pseudo-Boolean algebra with respect to $\cap, \cup, \supset$ and 0 . Moreover, we have that for any $a$ in $P_{0}$ $I a$ and $C a$ are also in $P_{0}$. When we prove that $C a \in P_{0}$, we represent the element $a$ in the disjunctive form and use (7). Let $I_{0}$ and $C_{0}$ be the restrictions of $I$ and $C$, respectively, to $P_{0}$. It remains to us to show that the set of clopen elements in $\left(P_{0}, I_{0}, C_{0}\right)$ constitutes a subalgebra of $P_{0}$. It is sufficient to prove that for every clopen $a, b \in P_{0}, a \supset b$ is also clopen, since other cases are obvious. By the definition of $\supset$, let $a \supset b=\cup_{i} z_{i}$, where $\left\{z_{i}\right\}_{i}$ is an enumeration of such an element $z$ of $P_{0}$ that $z \leq a \supset b$. It is obvious that $a \supset b \leq \bigcup_{i} C z_{i}$. On the other hand, $C z_{j}$ 
$\leq C(a \supset b)=a \supset b$ for each $j$, since both $a$ and $b$ are clopen in the bi-tpba $(P, I, C)$ of type 32 . Thus, for each $j C z_{j}$ must be in $\left\{z_{i}\right\}_{i}$. Hence $\cup_{i} C z_{i} \leq a \supset b$. So,

$$
a \supset b=\bigcup_{i} C z_{i}=C\left(\bigcup_{i} z_{i}\right)=C_{0}\left(\bigcup_{i} z_{i}\right) .
$$

Hence, $a \supset b$ is clopen. So, similarly as the proof of Theorein 4. 3, we can show that $A$ is not valid in $\left(P_{0}, I_{0}, C_{0}\right)$.

Corollary 4.7. $L_{32}$ has the finite model propertv and hence is decidable.

Proof. Let $A$ be a formula not containing $\diamond$, which is not provable in $\mathrm{L}_{32}$. Then $A$ is not provable also in $\mathrm{L}_{32}$ by Theorem 4. 4. By Theorem 4.6, there exists a finite bi-tpba $\left(P_{0}, I_{0}, C_{0}\right)$ of type 32 and its assignment $g$ such that $g(A) \neq 1$. Then, $\left(P_{0}, I_{0}\right)$ is a finite tpba of type 32 . Let $h$ be an assignment of $\left(P_{0}, I_{0}\right)$, which is obtained from $g$ by restricting the domain of $g$ to the set of formulas not containing $\diamond$. Then, clearly $h(A) \neq 1$.

Since a tpba $(P, I)$ of type 2 does not always satisfy the condition $I(I a \cup b) \leq I a \cup I b$, we must alter the above proof slightly to show the finite model property for $\mathrm{L}_{2}$.

Theorem 4. 8. $L_{2^{*}}$ has the finite model property and hence is decidable. ${ }^{1)}$

Proof. Let $A$ be a formula not provable in $\mathrm{L}_{2^{2}}$. Then, there is a bi-tpba $(P, I, C)$ of type 2 and its assignment $f$ such that $f(A) \neq 1$. Take the elements $a_{1}, \cdots, a_{m}$, as in the proof of Theorem 4.6. Let $S$ be the set defined by

$$
S=\left\{0,1, a_{1}, \cdots, a_{m}\right\} \cup\left\{C U_{*} ; U \text { is a nonempty subset of }\left\{a_{1}, \cdots, a_{m}\right\}\right\}
$$

The sublattice $P_{0}$ generated by $S$ is a pseudo-Boolean algebra with respect

1) Bull showed in [1] that $\mathrm{L}_{2^{*}}$ has the finite model property. But his proof seems to contain a gap. 
to $\cap, \cup, \supset$ and 0 . As Theorem 4.6, if $a \in P_{0}$ then $C a \in P_{0}$. Let $C_{0}$ be the restriction of $C$ to $P_{0}$. Now define an operation $I^{\prime}$ on $P_{0}$ by $I^{\prime} a=\bigcup_{z}\left\{z ; z \in P_{0}, z\right.$ is clopen in $(P, I, C)$ and $\left.z \leq a\right\}$, for any $a \in P_{0}$. Since $P_{0}$ is finite and is closed with respect to the join, $I^{\prime} a$ is also in $P_{0}$. We show that $\left(P_{0}, I^{\prime}, C_{0}\right)$ is a bi-tpba of type 2 . First we prove that $\left(P_{0}, I^{\prime}, C_{0}\right)$ is a bi-tpba.

$$
I^{\prime}(a \cap b)=I^{\prime} a \cap I^{\prime} b .
$$

It is obvious that $I^{\prime}(a \cap b) \leq I^{\prime} a \cap I^{\prime} b$. Let $I^{\prime} a=\cup z_{\text {, }}$ and $I^{\prime} b=\cup w_{j}$, where both $z$, and $w_{1}$ are clopen, $z_{i} \leq a$ and $w_{j} \leq b$. 'Then

$$
I^{\prime} a \cap I^{\prime} b=\bigcup_{i} z_{i} \cap \bigcup_{j} w_{j}=\bigcup_{i, j}\left(z_{1} \cap w_{j}\right) \text {. }
$$

Since $z_{i} \cap \mathfrak{r}_{j}^{\prime}$ is clopen and $z_{i} \cap w_{j} \leq a \cap b$ for each $i, j, \underset{i, j}{\cup}\left(z_{i} \cap w_{j}\right) \leq$ $I^{\prime}(a \cap b)$. Thus, $I^{\prime} a \cap I^{\prime} b \leq I^{\prime}(a \cap b)$.

$$
C_{0} I^{\prime} a=I^{\prime} a \text {. }
$$

Let $I^{\prime} a=\bigcup_{i} z_{i}$, where each $z_{i}$ is clopen and satisfies $z_{i} \leq a$. Then

$$
C_{0} I^{\prime} a=C_{0}\left(\bigcup_{i} z_{i}\right)=\bigcup_{i} C_{0} z_{i}=\bigcup_{i} z_{i}=I^{\prime} a
$$

Other conditions are easily verified. It is easy to see that if an element is clopen in $\left(P_{0}, I^{\prime}, C_{0}\right)$ then it is also clopen in $(P, I, C)$. Using this, we can show similarly as Theorem 4.6 that $\left(P_{0}, I^{\prime}, C_{0}\right)$ is of type 2 . Let $g$ be an assignment of $\left(P_{0}, I^{\prime}, C_{0}\right)$ such that $g(p)=f(p)$ for any propositional variable $p$ appearing in $A$. Then we have that $g(A)=f(A)$ $\neq 1$. Notice that $I a \in P_{0}$ implies $I^{\prime} a=I a$. Hence, $A$ is not valid in $\left(P_{0}\right.$, $\left.I^{\prime}, C_{0}\right)$.

Now we can prove the following corollary quite similarly as Corollary 4. 7.

Corollary 4.9. $L_{2}$ has the finite model property and hence is decidable.

Modifying the proof of Theorem 4.8, we can get another proof of Theorem 4.1. In proving Corollaries 4.7 and 4.9, we have first shown the finite model property for a conservative extension and then derived 
the finite model property for the IML under consideration. We have no direct proofs at present. For $\mathrm{L}_{1}$ and $\mathrm{L}_{31}$, we can define $\mathrm{L}_{1^{*}}$ and $\mathrm{L}_{31^{*}}$ by adding the axioms from (1) to (6). Then we can also prove that both $\mathrm{L}_{1 *}$ and $\mathrm{L}_{31}$, have the finite model property. But we don't know whether they are conservative extension of $\mathrm{L}_{1}$ and $\mathrm{L}_{31}$, respectively.

\section{§ 5. M Models}

In this section, we will introduce another type of Kripke models, called $M$ models. A triple $(M, R, P)$ is an $M$ frame, if

1) $M$ is a nonempty set,

2) $R$ is a reflexive and transitive relation on $M$,

3) $P$ is a non-degenerate $\kappa(M)^{<}$-complete pseudo-Boolean algebra, where $\kappa(M)$ is the smallest cardinal which is greater than $\overline{\overline{\{b ; a R b\}}}$ for any $a \in M$. More precisely, for any subset $\left\{a_{t}\right\}_{t \in T}$ of $P$ such that $\overline{\bar{T}}<\kappa(M)$, there exist $\bigcup_{t \in T} a_{t}$ and $\bigcap_{t \in T} a_{t}$ in $P$.

A valuation $W$ on an $M$ frame $(M, R, P)$ is a mapping from the direct product of the set formulas and the set $M$ to the set $P$ such that for any $a \in M$

$$
\begin{aligned}
& \text { 1) } \quad W(A \wedge B, a)=W(A, a) \cap W(B, a), \\
& \text { 2) } \quad W(A \vee B, a)=W(A, a) \cup W(B, a), \\
& \text { 3) } \quad W(A \supset B, a)=W(A, a) \supset W(B, a), \\
& \text { 4) } \quad W(\neg A, a)=-W(A, a), \\
& \text { 5) } \quad W(\square A, a)=\bigcap_{a R b} W(A, b) .
\end{aligned}
$$

In the above definition, $\cap, \cup, \supset$ and -, which appear in the right side of equations, denote lattice operations in $P$. A quadruple $(M, R, P, W)$ is an $M$ model if $(M, R, P)$ is an $M$ frame and $W$ is a valuation on it. A formula $A$ is valid in an $M$ model $(M, R, P, W)$ if $W(A, a)=1$ for any $a$ in $M$, where 1 is the greatest element of $M$. $A$ is valid in an $M$ frame $(M, R, P)$ if it is valid in an $M$ model $(M, R, P, W)$ for any valuation $W$. We can verify the following lemma.

Lemma 5. 1. Every formula provable in $L_{0}$ is valid in any $M$ 
model.

Let us define an $M$ frame of type $J$, where $J=0,2,32$.

0) Any $M$ frame is of type 0 .

1) An $M$ frame $(M, R, P)$ is of type 2, if $R$ is symmetric (and hence is an equivalence relation).

2) An $M$ frame $(M, R, P)$ is of type 32, if it is of type 2 and satisfies the following distributive law: For any subset $\left\{a_{t}\right\}_{t \in T}$ of $P$ such that $\overline{\bar{T}}<\kappa(M)$ and for any element $b$ of $P$,

$$
\bigcap_{i \subset T}\left(a_{t} \cup b\right) \leq \bigcap_{t \in T} a_{\imath} \cup b .
$$

An $M$ model $(M, R, P, W)$ is said to be of type $J$ if the $M$ frame $(M, R, P)$ is of type $J$.

Lemma 5. 2. For any $M$ frame $(M, R, P$ ) of type 2 (or of type 32), there exists a set $\left\{\left(M_{i}, R_{i}, P\right)\right\}_{i}$ of $M$ frames of type 2 (or of type 32 , respectively) such that

1) for any $i$ and any $x, y \in M_{i}, x R_{i} y$,

2) a formula is valid in $(M, R, P)$ if and only if it is valid in any $\left(M_{i}, R_{i}, P\right)$.

Proof. Let $\left\{M_{i}\right\}_{i}$ be the set of all equivalence class determined by the equivalence relation $R$. Let $R_{i}$ be the restriction of $R$ to $M_{i}$. Then 1) and 2) are easily verified.

As proved in Theorem 2.1, $\mathrm{L}_{2}$ and $\mathrm{L}_{32}$ are equivalent to sequent calculi $G_{2}$ and $G_{32}$, respectirely. Let $G_{2^{*}}$ be the sequent calculus obtained from $G_{2}$ by adding the following rules of inference concerning $\diamond$.

$$
(\diamond \rightarrow) \frac{A, \Gamma \rightarrow \Delta}{\diamond A, \Gamma \rightarrow \Delta}(\rightarrow \diamond) \frac{\Gamma \rightarrow A}{\Gamma \rightarrow \diamond A},
$$

where $\Delta$ consists of at most one formula. In the application of $(\diamond \rightarrow)$ rule, we impose the condition that both $\Gamma$ and $\Delta$ are sequences of $b i$ completely modalized formulas, where a formula is said to be bi-completely modalized if any occurrence of a propositional variable in it is within the scope of a model operator (i.e. either $\square$ or $\diamond$ ). Let $G_{32^{+}}$ 
be the sequent calculus obtained from $\mathrm{G}_{32}$ by adding the following rules of inference.

$$
(\diamond \rightarrow+) \frac{A, \Gamma \rightarrow \Delta}{\diamond A, \Gamma \rightarrow \Delta}(\rightarrow \diamond+) \frac{\Gamma \rightarrow \Delta, A}{\Gamma \rightarrow \Delta, \diamond A} .
$$

In this case, $\Delta$ may contain an arbitrary number of formulas. In the application of $(\diamond \rightarrow+)$, we also impose the same condition as that of $\mathrm{G}_{2^{*}}$. Now we have the following theorem.

Theorem 5.3. $L_{2^{+}}\left(\right.$or $\left.L_{32^{+}}\right)$is equivalent to $G_{2^{+}}$(or $G_{32^{+}}$, respectively).

Let $\mathrm{LD}$ be the sequent calculus obtained from $\mathrm{LJ}^{\prime}$ by replacing the rule $(\rightarrow \forall)$ by the rule

$$
\frac{\Gamma \rightarrow \Delta, A(a)}{\Gamma \rightarrow \Delta, \forall x A(x)}
$$

where $a$ is a variable not appearing in the lower sequent (see [22]). It is known that 1) LD is equivalent to the intuitionistic predicate logic with the axiom schema $\forall x(A(x) \bigvee B) \supset(\forall x A(x) \vee B)$, where $x$ is a variable not occurring free in $B$, and 2) $\mathrm{LD}$ is complete with respect to Kripke models with a constant domain (see [7]). It seems that there exist close connections between $G_{2^{*}}$ and $L J$ and between $G_{32^{*}}$ and $L D$. For, if we interprete $\square$ and $\diamond$ as the universal and the existential quantifier and regard the condition of the bi-complete modalization as the variable condition in the application of rules of $\mathrm{G}_{2^{*}}$ and $\mathrm{G}_{32^{*}}$, then we have the corresponding systems LJ and LD. These connections are also found out in the definition of $I$ frames of type 2 or type 32 . The set $\{b ; a \sim b\}$ for any element $a$ in an $I$ frame corresponds to the domain attached to $a$ of a Kripke frame. Now, let us state these connections more precisely. For any variable $x$ we define a translation $\psi_{x}$ from the set of formulas of modal propositional logics to the set of formulas of predicate logics. First, we take a monadic predicate variable $P(x)$ for each propositional variable $p$. Then we define that

$$
\psi_{x}(p)=P(x) \text { if } p \text { is a propositional variable, }
$$




$$
\begin{aligned}
& \psi_{x}(A \wedge B)=\psi_{x}(A) \wedge \psi_{x}(B), \\
& \psi_{x}(A \vee B)=\psi_{x}(A) \vee \psi_{x}(B), \\
& \psi_{x}(A \supset B)=\psi_{x}(A) \supset \psi_{x}(B), \\
& \psi_{x}(\neg A)=\neg \psi_{x}(A), \\
& \psi_{x}(\square A)=\forall x \psi_{x}(A), \\
& \psi_{x}(\diamond A)=\exists x \psi_{x}(A) .
\end{aligned}
$$

It is well-known that a formula $A$ is provable in S5 if and only if $\psi_{x}(A)$ is provable in $\mathrm{LK}$.

Theorem 5. 4. 1) (Bull [3]) A formula $A$ is provable in $G_{2^{*}}$ if and only if $\psi_{x}(A)$ is provable in LJ.

2) A formula $A$ is provable in $G_{32^{*}}$ if and only if $\psi_{x}(A)$ is provable in $L D$.

Proof of 2). We prove 2) in the same way as [3]. Only if part is obvious. We first replace every occurrence of $\psi(t)$ by a constant $V$, in the definition of Kripke's semantic tableaux [12]. Then we replace the rule $\Pi_{r}$ by the following $\Pi_{r}{ }^{*}$.

$\Pi_{r}{ }^{*}$. If $\forall x A(x)$ appears on the right of a tableau $t$, and $y$ is the alphabetically earliest variable which has not yet appeared in any tableau of any alternative set at this stage, put $y \in V$ and put $A(y)$ on the right of $t$.

Then we can show that the following three conditions are equivalent.

1) The construction for A thus modified is closed.

2) A is valid in any Kripke model with a constant domain.

3) $A$ is provable in $L D$.

Now, using the normal construction as [3], we have our theorem.

It should be remarked that $\mathrm{G}_{2^{*}}$ is not cut-free, while $\mathrm{LJ}$ is cut-free. This remark can also apply to the sequent calculus S5 in [15] and LK. But as Kanger did for S5 in [10], we can construct a cut-free system for $\mathrm{L}_{2^{*}}$ by using Theorem 5. 4 .

Next we show the completeness theorem with respect to $M$ models. 
Lemma 5. 5. For any formula $A$, $A$ is valid in any $M$ model. of type 2 if and only if $\psi_{x}(A)$ is provable in LJ.

Proof. We first notice that $A$ is valid in any $M$ model of type 2 if and only if $\square A$ is valid in any $M$ model of type 2 and that for any formula $B$ of predicate logics, $B$ is provable in LJ if and only if any universal closure of $B$ is provable in LJ. So, it is sufficient to prove the lemma for the case where $A$ is of the form $\square B$, since $\psi_{x}(\square B)$ $=\forall x \psi_{x}(B)$. Now suppose that $\psi_{x}(A)$ is not provable in LJ. Then, there exists a pseudo-Boolean frame $(P, V)$ consisting of a complete pseudo-Boolean algebra $P$ and a domain $V$ such that for some assignment $f$ $f\left(\psi_{x}(A)\right) \neq 1$ in $(P, V)$. (See [19]. In [16], a pseudo-Boolean frame is called as a pseudo-Boolean model.) Let $R$ be a relation on $V$ such that $v R w$ holds for every $v, w \in V$. Then, $(V, R, P)$ is an $M$ frame of type 2. Define a valuation $W$ on $(V, R, P)$ by $W(p, v)=f(P(\bar{v}))$ for any propositional variable $p$ and any $v \in V$, where $\bar{v}$ denotes the name of $v$. Then it is easily seen that for any formula $C$ of modal logics, $W(C, v)=f\left(\mathrm{~S}_{\bar{v}}^{x} \psi_{x}(C)\right)$, where $\mathrm{S}_{\bar{v}}^{x}$ means the substitution of $\bar{v}$ for any free occurrence of a variable $x$. Then for any $v \in V, W(A, v)=$ $f\left(\mathrm{~S}_{\bar{v}}{ }^{x} \psi_{x}(A)\right)=f\left(\psi_{x}(A)\right) \neq 1$, since $\psi_{x}(A)\left(=\forall x \psi_{x}(B)\right)$ is a closed formula by our assumption. Hence $A$ is not valid in $(V, R, P, W)$. Conversely, suppose that $A$ is not valid in an $M$ model $(M, R, P, W)$ of type 2 . By Lemma 5. 2, we can assume that $x R y$ holds for any $x, y \in M$. In this case, $\kappa(M)^{<}$-completeness of $P$ means $\overline{\bar{M}}$-completeness. So, $(P, M)$ is a pseudo-Boolean frame for LJ. Define an assignment $f$ of $(P, M)$ by $f(P(\bar{v}))=W(p, v)$ for any propositional variable $p$ and any $v \in M$. Then, as the above, we have $f\left(\psi_{x}(A)\right)=W(A, v)$ for any $v \in M$. So, $f\left(\psi_{x}(A)\right) \neq 1$ by the assumption. Hence, $\psi_{x}(A)$ is not provable in LJ.

Lemma 5. 6. For any formula $A, A$ is valid in any $M$ model of type 32 if and only if $\psi_{x}(A)$ is provable in LD.

This lemma can be proved similarly as Lemma 5.5. In the proof, we use the completeness theorem of $\mathrm{LD}$ with respect to complete pseudoBoolean frames satisfying (1) (see [7]). 
Theorem 5. 7. A formula is provable in $L_{2}\left(\right.$ or $\left.L_{32}\right)$ if and only if it is valid in any $M$ model of type 2 (or type 32, respectively).

Proof. By Lemma 5.5 (or 5.6), a formula $A$ is valid in any $M$ model of type 2 (or type 32 ) if and only if $\psi_{x}(A)$ is provable in LJ (or LD). Then by Theorems 5.3 and 5.4, this is equivalent to the condition that $A$ is provable in $\mathrm{L}_{2^{+}}$(or $\mathrm{L}_{32^{+}}$). By Lemma 4.4, this is also equivalent to the condition that $A$ is provable in $\mathrm{L}_{2}\left(\right.$ or $\left.\mathrm{L}_{32}\right)$.

Now, recall the condition (1). If the set $T$ is finite then (1) holds always. Thus, any formula provable in $\mathrm{L}_{32}$ is valid in any $M$ model $(M, R, P, W)$ of type 2 with a finite set $M$. Moreover, the following lemma holds.

Lemma 5. 8. Let $(M, R, P)$ be any $M$ frame. If either $A_{1}$ or $A_{3}$ is valid in $(M, R, P)$ then $R$ is symmetric.

Proof. Suppose that $A_{1}$ (i.e., $\left.\neg \square p \supset \square \neg \square p\right)$ is valid in $(M, R, P)$ and that $R$ is not symmetric. Then there are $a, b \in M$ such that $a R b$ but not $b R a$. Let $S$ and $T$ be subsets of $M$ defined by

$$
\begin{aligned}
& S=\{x ; a R x \text { but not } x R a\}, \\
& T=\{x ; a R x \text { and } x R a\} .
\end{aligned}
$$

Then for any $x$ such that $a R x$, either $x \in S$ or $x \in T$. Since $b \in S$ and $a \in T$, both $S$ and $T$ are not empty. Define a valuation $W$ on $(M, R, P)$ by

$$
W(p, x)= \begin{cases}1 & \text { if } x \in S \\ 0 & \text { if } x \in T\end{cases}
$$

Since $a \in T$ and $a R a, \bigcap_{a R x} W(p, x) \leq W(p, a)=0$. Thus, $W(\neg \square p, a)$ $=-\bigcap_{a R x} W(p, x)=1$. On the other hand,

$$
\begin{aligned}
W(\square \neg \square p, a) & =\bigcap_{a R x}-\bigcap_{x R y} W(p, y) \\
& =\left(\bigcap_{x \in S}-\bigcap_{x R y} W(p, y)\right) \cap\left(\bigcap_{x \in T}-\bigcap_{x R y} W(p, y)\right) .
\end{aligned}
$$


It is easy to see that if $x \in S$ and $x R y$ then $y \in S$. So,

$$
\bigcap_{x \in S}-\bigcap_{x R_{y}} W(p, y)=\bigcap_{x \in S}-1=0 .
$$

Thus $W(\square \neg \square p, a)=0$. Hence $W(\neg \square p \supset \square \neg \square p, a)=0$. But this contradicts the assumption. Hence $R$ is symmetric. The case where $A_{3}$ is valid in $(M, R, P)$ can be treated similarly.

Using the above lemma, we have the following theorem (cf. Corollary 4.9.). Here we say that an IML $L$ is characterized by a set of $M$ frames $\left(\left\{M_{i}, R_{i}, p_{i}\right)\right\}_{i}$, if it holds that for any formula $A, A$ is provable in $\mathrm{L}$ if and only if $A$ is valid in any $\left(M_{i}, R_{i}, P_{i}\right)$.

Theorem 5.9. $L_{2}$ can not be characterized by any set of $M$ frames $(M, R, P)$, where each $M$ is finite. Hence, $L_{2}$ has not the finite model property with respect to $M$ frames.

Similarly, we can answer the question presented by Bull in [1] negatively.

Theorem 5. 10. $L_{32}$ can not be characterized by any set of $M$ frames $(M, R, P)$, where each $M$ is finite. Hence, $L_{32}$ has not the finite model property with respect to $M$ frames.

Proof. Let $A$ be the formula $\square \neg \neg p \supset \neg \neg \square p$. We show first that $A$ is not provable in $\mathrm{L}_{32}$. Define an I model $\left(M, \leq^{*}, R, W\right)$ as follows;

1) $M$ is the set $N \times N$, where $N$ is the set of natural numbers,

2) for every $i, j, m, n \in N$,

$$
\langle i, m\rangle \leq^{*}\langle j, n\rangle \Leftrightarrow i \leq j \text { and } m=n,
$$

and

$$
\langle i, m\rangle R\langle j, n\rangle \Leftrightarrow i \leq j,
$$

3) for every $i, m \in N$,

$$
W(p,\langle i, m\rangle)=t \Leftrightarrow i>m .
$$


Clearly, the $I$ model thus defined is of type 32 . Moreover, we can show that $W(A,\langle 0,0\rangle)=f$. Thus, $A$ is not provable in $\mathrm{L}_{32}$ by Theorem 3.2. Next we show that $A$ is valid in any $M$ frame $(M, R, P)$ with a finite set $M$, in which axioms of $\mathrm{L}_{32}$ are valid. By Lemma $5.8, R$ is symmetric. By Lemma 5. 2, we can also assume that for every $x, y \in M, x R y$ holds. Let $W$ be any valuation on $(M, R, P)$. Then for any $a \in M$,

$$
W(A, a)=\left(\bigcap_{x \in M}--W(p, x)\right) \supset\left(--\bigcap_{x \in M} W(p, x)\right) .
$$

We write $u_{r}$ for $W^{\top}(p, x)$. We show by induction on the number $n$ of elements in $M /$ that

$$
\bigcap_{r \in M}--u_{x} \leq--\bigcap_{x \in M} u_{r}
$$

Clearly (2) holds if $n=1$. Consider the case where $n=k+1$. Let $M I^{\prime}$ $=M I-\{a\}$ and $v=\bigcap_{x \equiv M^{\prime}} u_{x}$. Since $\overline{\bar{\Lambda}} I^{\prime}=k<n$, by using the induction hypothesis,

$$
\begin{aligned}
\bigcap_{x \in M}--u_{x} & =\bigcap_{x \subset M^{\prime}}--u_{x} \cap--u_{a} \\
& \leq--\bigcap_{x \in M^{\prime}} u_{x} \cap--u_{a} \\
& =--w \cap--u_{a} .
\end{aligned}
$$

On the other hand,

$$
w \cap\left(u_{a} \cap-\left(w \cap u_{a}\right)\right)=\left(w \cap u_{a}\right) \cap-\left(w \cap u_{a}\right) \leq 0 .
$$

Thus, $u_{a} \cap-\left(w \cap u_{a}\right) \leq-w$. This implies that

$$
u_{a} \cap-\left(w \cap u_{a}\right) \cap--w \leq 0 .
$$

So, $-\left(w \cap u_{a}\right) \cap--w \leq-u_{a}$ and hence

$$
-\left(w \cap u_{a}\right) \cap--w \cap---u_{a} \leq 0 .
$$

Thus, $\bigcap_{x \in M}--u_{s} \leq--w \cap--u_{n}$

$$
\leq--\left(w \cap u_{a}\right)=--\bigcap_{x \in M} u_{x}
$$

Hence $W(A, a)=1$ for any $a \in M$. Therefore, $A$ is valid in any $M$ frame $(M, R, P)$ with a finite set $M$. Thus, $\mathrm{L}_{32}$ can not be characterized by any set of $M$ frames $(M, R, P)$ with a finite set $M$. 
Using Lemma 5. 8, we prove that other IML's except $\mathrm{L}_{0}$ are incomplete with respect to $M$ frames.

Theorem 5. 11. Any one of $L_{1}, L_{3}$ and $L_{31}$ is not complete with respect to any set of $M$ frames.

Proof. Let $\mathrm{L}$ be any one of $\mathrm{L}_{1}, \mathrm{~L}_{3}$ and $\mathrm{L}_{31}$. Suppose that $\mathrm{L}$ is characterized by a set of $M$ frames $\left\{\left(M_{i}, R_{i}, P_{i}\right)\right\}_{i}$. Since either $A_{1}$ or $A_{8}$ is provable in $\mathrm{L}$, each $R_{i}$ is symmetric by Lemma 5.8. Then each $\left(M_{i}, R_{i}, P_{i}\right)$ is of type 2 and hence $\mathrm{L}$ must be equal to or stronger than $\mathrm{L}_{2}$ by Theorem 5. 7. But this contradicts the inclusion relationship mentioned in $\S 2$.

Theorem 5. 12. $L_{4}$ is not complete with respect to any set of $M$ frames.

Proof. Suppose that $\mathrm{L}_{4}$ is characterized by a set of $M$ frames $\left\{\left(M_{i}\right.\right.$, $\left.\left.R_{i}, P_{i}\right)\right\}_{i}$. Since $A_{1}$ is provable in $\mathrm{L}_{4}$, each $R_{i}$ is symmetric. Moreover, by using Lemma 5.2 , we can assume that for each $i, x R_{i} y$ holds for every $x, y \in M_{i}$. Now for a fixed $i$ take any element $u$ in $P_{i}$. Define a valuation $W_{i}$ on $\left(M_{i}, R_{i}, P_{i}\right)$ by $W_{i}(p, a)=u$ for any $a \in M_{i}$. Then,

$$
\begin{aligned}
W_{i}(\square p \vee & \square \neg \square p, a) \\
& =\bigcap_{a R_{i} b} W_{i}(p, b) \cup \underset{a R_{i} b}{\cap}-\bigcap_{a R_{i} c} W_{i}(p, c) \\
& =u \cup-u .
\end{aligned}
$$

Since $\square p \vee \square \neg \square p$ is provable in $\mathrm{L}_{4}$,

$$
W_{i}(\square p \vee \square \neg \square p, a)=u \cup-u=1 \text {. }
$$

This means that each $P_{i}$ is a Boolean algebra. Thus, $A \bigvee \neg A$ is valid in any $\left(M_{i}, R_{i}, P_{i}\right)$ and hence is provable in $\mathrm{L}_{4}$. But this is a contradiction.

We don't know whether $\mathrm{L}_{0}$ is complete with respect to $M$ frames of type 0 or not.

Finally, we remark a connection between $M$ frames and tpba's. Let 
$(M, R, P)$ be an $M$ frame. Then the set $P^{M}$ of all functions from $M$ to $P$ constitutes a pseudo-Boolean algebra. More precisely, for any $f, g$ in $P^{M}$, define $f \cap g, f \cup g$ and $f \supset g$ by

$$
\begin{aligned}
& (f \cap g)(a)=f(a) \cap g(a), \\
& (f \cup g)(a)=f(a) \cup g(a), \\
& (f \supset g)(a)=f(a) \supset g(a)
\end{aligned}
$$

for any $a \in M$. Let 0 be a function in $P^{M}$ such that $0(a)=0_{P}$ for any $a \in \Lambda$, where $0_{P}$ is the least element of $P$. Then $P^{M}$ with $\cap, \cup, \supset$ and 0 is a pseudo-Boolean algebra. Next, define a unary operation $I_{R}$ on $P^{M}$ by

$$
\left(I_{R} f\right)(a)=\bigcap_{a R b}(f(b))
$$

for any $a \in M$. Then $\left(P^{M}, I_{R}\right)$ becomes a tpba. Now suppose that for any propositional variable $p$ and any $a \in M$, it holds that $W(p, a)$ $=\varphi(p)(a)$, where $W$ is a valuation on $(M, R, P)$ and $\varphi$ is an assignment of $\left(P^{M}, I_{R}\right)$. Then we can show that for any formula $A, W(A, a)$ $=\varphi(A)(a)$. From this it follows that for any formula $A, A$ is valid in $(M, R, P)$ if and only if $A$ is valid in $\left(P^{M}, I_{R}\right)$.

\section{References}

[1] Bull, R. A., A modal extension of intuitionistic logic, Notre Dame J. of Formal Logic, 6 (1965), 142-146.

[2] Some modal calculi based on IC, Formal systems and recursive functions, ed. by J. N. Crossley and M. A. E. Dummett, Amsterdam, 1965, 3-7.

[3] , MIPC as the formalisation of an intuitionist concept of modality, J. Symbolic Logic, 31 (1966), 609-616.

[4] Fitch, F. B., Intuitionistic modal logic with quantifiers, Portugaliae Mathematica, 7 (1948), 113-118.

[5] Fitting, M., Model existence theorems for modal and intuitionistic logics, J. Symbolic Logic, 38 (1973), 613-627.

[6] Gentzen, G., Untersuchungen über das logische Schliessen, I. II., Math. Z., 39 (1935), 176-210, 405-431.

[7] Görnemann, S., A logic stronger than intuitionism, J. Symbolic Logic, 36 (1971), 249-261.

[8] Ishimoto, A. and Watanabe, Y., Some bi-modal propositional logics and their completeness, Bulletin of Tokyo Institute of Technology, 124 (1974), 63-79.

[9] Jankov, V. A., Constructing a sequence of strongly independent superintuitionistic propositional calculi, Soviet Math. Dokl., 9 (1968), 806-807.

[10] Kanger, S., Provability in logic, Stockholm Studies in Philosophy 1, Stockholm, 1957.

[11] Kripke, S. A., Semantical analysis of modal logic I, Z. Math. Logik Grundlagen 
Math., 9 (1963), 67-96.

[t2] - - Semantical analysis of intuitionistic logic I, Formal systems and recursive functions, ed. by J. N. Crossley and M. A. Dummett, Amsterdam, 1965, 92-130.

[13] Lemmon, E. J., Algebraic semantics for modal logics I, J. Symbolic Logic, 31 (1966), 46-65

[14] McKinsey, J. C. C. and Tarski, A., Some theorems about the sentential calculi of Lewis and Heyting, J. Symbolic Logic, 13 (1948), 1-15.

[15] Ohnishi, M. and Matsumoto, K., Gentzen method in modal calculi, Osaka Math. J., 9 (1957), 113-130.

[16] Ono, H., A study of intermediate predicate logics, Publ. RIMS, Kyoto Univ., 8 (1973), 619-649.

[17] Prawitz, D., Natural deduction, A proof theoretical study, Stockholm studies in philosophy 3, Stockholm, 1965.

[18] Prior, A. N., Time and modality, Oxford, 1957.

[19] Rasiowa, H. and Sikorski, R., The mathematics of metamathematics, Monografie Matematyczne, 41, Warszawa, 1963.

[20] Sato, M., A study of Kripke-type models for some modal logics by Gentzen's sequential method, Publ. RIMS, Kyoto Univ., 13 (1977), 381-468.

[21] Schütte, K., Vollständige Systeme modaler und intuitionistischer Logik, Ergebnisse der Mathematik und ihrer Grenzgebiete, Band 42, 1968.

[22] Umezawa, T., On logics intermediate between intuitionistic and classical predicate logic, J, Symbolic Logic, 24 (1959), 141-153. 